\title{
Induced-gravity GUT-scale Higgs inflation in supergravity
}

\author{
Constantinos Pallis ${ }^{1, a}$, Qaisar Shafi, ${ }^{2, b}$ \\ ${ }^{1}$ School of Electrical and Computer Engineering, Faculty of Engineering, Aristotle University of Thessaloniki, 54124 Thessaloniki, Greece \\ ${ }^{2}$ Department of Physics and Astronomy, Bartol Research Institute, University of Delaware, Newark, DE 19716, USA
}

Received: 4 March 2018 / Accepted: 7 June 2018 / Published online: 26 June 2018

(C) The Author(s) 2018

\begin{abstract}
Models of induced-gravity inflation are formulated within Supergravity employing as inflaton the Higgs field which leads to a spontaneous breaking of a $U(1)_{B-L}$ symmetry at $M_{\mathrm{GUT}}=2 \cdot 10^{16} \mathrm{GeV}$. We use a renormalizable superpotential, fixed by a $U(1) \mathrm{R}$ symmetry, and Kähler potentials which exhibit a quadratic non-minimal coupling to gravity with or without an independent kinetic mixing in the inflaton sector. In both cases we find inflationary solutions of Starobinsky type whereas in the latter case, others (more marginal) which resemble those of linear inflation arise too. In all cases the inflaton mass is predicted to be of the order of $10^{13} \mathrm{GeV}$. Extending the superpotential of the model with suitable terms, we show how the MSSM $\mu$ parameter can be generated. Also, non-thermal leptogenesis can be successfully realized, provided that the gravitino is heavier than about $10 \mathrm{TeV}$.
\end{abstract}

\section{Introduction}

The idea of induced gravity (IG), according to which the (reduced) Planck mass $m_{\mathrm{P}}$ is generated [1,2] via the vacuum expectation value ( v.e.v) that a scalar field acquires at the end of a phase transition in the early universe, has recently attracted a fair amount of attention. This is because it may follow an inflationary stage driven by a Starobiskytype potential [3] in Supergravity (SUGRA) [4-13] and in non-Supersymmetric (SUSY) [14-22] settings, which turns out to be nicely compatible with the observational data [23]. As a bonus, the resulting effective theories do not suffer from any problem with perturbative unitarity $[4,5,7-9,20,24-26]$ in sharp contrast to some models of non-minimal inflation [27-31] where the inflaton after inflation assumes a v.e.v much smaller than $m_{\mathrm{P}}$.

\footnotetext{
a e-mail: kpallis@gen.auth.gr

be-mail: shafi@bartol.udel.edu
}

The simplest way to realize the idea of IG is to employ a double-well potential, $\lambda\left(\phi^{2}-v^{2}\right)^{2}$, for the inflaton $\phi[1,2,4$ 9,14-20] - scale invariant realizations of this idea are proposed in Refs. [21,22]. If we adopt a non-minimal coupling to gravity [17-19] of the type $f_{\mathcal{R}}=c_{\mathcal{R}} \phi^{2}$ and set $v=m_{\mathrm{P}} / \sqrt{c_{\mathcal{R}}}$, then $\left\langle f_{\mathcal{R}}\right\rangle=m_{\mathrm{P}}^{2}$, i.e., $f_{\mathcal{R}}$ reduces to $m_{\mathrm{P}}^{2}$ at the vacuum generating, thereby, Einstein gravity at low energies. The implementation of inflation, on the other hand, which requires the emergence of a sufficiently flat branch of the potential at large field values constrains $c_{\mathcal{R}}$ to sufficiently large values and $\lambda$ as a function of $c_{\mathcal{R}}$. An even more restrictive version of this scenario would be achieved if $\phi$ is involved in a Higgs sector which triggers a Grand Unified Theory (GUT) phase transition in the early Universe $[13,17,18]$. The scale of a such transition is usually related to the (field dependent) mass of the lightest gauge boson and can be linked to some unification condition in supersymmetric (SUSY) - most notably - settings [30-35]. As a consequence, $c_{\mathcal{R}}$ can be uniquely determined by the theoretical requirements, giving rise to an economical, predictive and well-motivated set-up, thereby called IG Higgs inflation (IGHI). To our knowledge, the unification hypothesis has not been previously employed in constraining IGHI.

Since gauge coupling unification is elegantly achieved within the minimal supersymmetric standard model (MSSM), we need to formulate IGHI in the context of SUGRA. Namely, we employ a renormalizable superpotential, uniquely determined by a gauge and a $U(1) \mathrm{R}$ symmetry, which realizes the Higgs mechanism in a SUSY framework. Actually, this is the same superpotential widely used for the models of F-term hybrid inflation [36-46]. Contrary to that case, though, where the inflaton typically is a gauge singlet and a pair of gauge non-singlets are stabilized at zero, here the inflaton is involved in the Higgs sector of the theory whereas the gauge singlet superfield is confined at the origin playing the role of a stabilizer - for a related scenario see Ref. [47]. For this reason we call it Higgs inflation (HI). As regards the Kähler potentials, $K$, we concentrate on 
semi-logarithmic ones which employ variable coefficients for the logarithmic part and include only quadratic terms of the various fields, taking advantage of the recently established [10-12] stabilization mechanisms of the accompanying noninflaton fields.

More specifically, we distinguish two different classes of $K$ 's, depending whether we introduce an independent kinetic mixing in the inflaton sector or not. In the latter case the nonminimal coupling to gravity reads $f_{\mathcal{R}} \sim c_{\mathcal{R}} \phi^{2}$ and imposing the IG and unification conditions allows us to fully determine $c_{\mathcal{R}}$. In the former case, apart from the non-minimal coupling to gravity expressed as $f_{\mathcal{R}}=c_{+} \phi^{2}$, the models exhibit a kinetic mixing of the form $f_{\mathrm{K}} \simeq c_{-} f_{\mathcal{R}}$, where the constants $c_{-}$and $c_{+}$can be interpreted as the coefficients of the principal shift-symmetric term $\left(c_{-}\right)$and its violation $\left(c_{+}\right)$in the $K$ 's. Obviously these models are inspired by the kinetically modified non-minimal HI studied in Refs. [32-35]. The observables now depend on the ratio $r_{ \pm}=c_{+} / c_{-}$which can be found precisely enforcing the IG and unification conditions. As a consequence, for both classes of models more robust predictions can be here achieved than those presented in the original papers [30-35], where $m_{\mathrm{P}}$ is included in $f_{\mathcal{R}}$ from every beginning. Most notably, the level of the predicted primordial gravitational waves is about an order of magnitude lower than the present upped bound $[23,59]$ and may be detectable in the next generation of experiments [60-63].

We exemplify our proposal employing as "GUT" gauge symmetry $G_{B-L}=G_{\mathrm{SM}} \times U(1)_{B-L}$, where $G_{\mathrm{SM}}=$ $S U(3)_{\mathrm{C}} \times S U(2)_{\mathrm{L}} \times U(1)_{Y}$ is the gauge symmetry of the standard model, and $B$ and $L$ denote baryon and lepton number respectively - cf. Refs. [32,34,35,43-45]. The embedding of IGHI within this particle model gives us the opportunity to connect inflation with low energy phenomenology. In fact, the absence of the gauge anomalies enforces the presence of three right-handed neutrinos $N_{i}^{c}$ which, in turn, generate the tiny neutrino masses via the type I seesaw mechanism. Furthermore, the out-of-equilibrium decay of the $N_{i}^{c}$ 's provides us with an explanation of the observed baryon asymmetry of the universe (BAU) $[64,65]$ via non-thermal leptogenesis (nTL) [66-69] consistently with the gravitino $(\widetilde{G})$ constraint [70-80] and the data [81-83] on the neutrino oscillation parameters. Also, taking advantage of the adopted $R$ symmetry, the parameter $\mu$ appearing in the mixing term between the two electroweak Higgs fields in the superpotential of MSSM is explained as in Refs. $[4,5,35,37]$ via the v.e.v of the stabilizer field, provided that the relevant coupling constant is appropriately suppressed. The post-inflationary completion induces more constraints testing further the viability of our models.

The remaining text is organized into three sections. We first establish and analyze our inflationary scenarios in Sect. 2. We then - in Sect. 3 - examine a possible postinflationary completion of our setting. Our conclusions are summarized in Sect. 4. Throughout the text, the subscript of type , $z$ denotes derivation with respect to ( w.r.t) the field $z$, and charge conjugation is denoted by a star. Unless otherwise stated, we use units where $m_{\mathrm{P}}=2.433 \cdot 10^{18} \mathrm{GeV}$ is taken to be unity.

\section{Inflationary models}

In Sect. 2.1 we describe the generic formulation of IG models within SUGRA, in Sect. 2.2, we construct the inflationary potential, and in Sect. 2.3 we analyze the observational consequences of the models.

\subsection{Embedding induced-gravity HI in SUGRA}

The implementation of IGHI requires the determination of the relevant super- and Kähler potentials, which are specified in Sect. 2.1.1. In Sect. 2.1.2 we present the form of the action in the two relevant frames and in Sect. 2.1.3 we impose the IG constraint.

\subsubsection{Set-up}

As we already mentioned, we base the construction of our models on the superpotential

$W_{\mathrm{HI}}=\lambda S\left(\bar{\Phi} \Phi-M^{2} / 4\right)$

which is already introduced in the context of models of F-term hybrid inflation [36]. Here $\bar{\Phi}, \Phi$ denote a pair of left-handed chiral superfields oppositely charged under $U(1)_{B-L} ; S$ is a $G_{B-L}$-singlet chiral superfield; $\lambda$ and $M$ are parameters which can be made positive by field redefinitions. $W_{\mathrm{HI}}$ is the most general renormalizable superpotential consistent with a continuous R symmetry [36] under which

$S \rightarrow e^{i \alpha} S, \bar{\Phi} \Phi \rightarrow \bar{\Phi} \Phi, W_{\mathrm{HI}} \rightarrow e^{i \alpha} W_{\mathrm{HI}}$.

Here and in the subsequent discussion the subscript $\mathrm{HI}$ is frequently used instead of IGHI to simplify the notation.

As we verify below, $W_{\mathrm{HI}}$ allows us to break the gauge symmetry of the theory in a simple, elegant and restrictive way. The v.e.vs of these fields, though, have to be related with the size of $m_{\mathrm{P}}$ according to the IG requirement. To achieve this, together with the establishment of an inflationary era, we have to combine $W_{\mathrm{HI}}$ with a judiciously selected Kähler potential, $K$. We present two classes of such $K$ 's, which respect the (gauge and global) symmetries of $W_{\mathrm{HI}}$ and incorporate only quadratic terms of the various fields. We distinguish these classes taking into account the origin of the kinetic mixing in the inflaton sector. Namely: 
(a) $K$ 's without independent kinetic mixing. Having in mind the general recipe [30,31,84-87] for the introduction of non-minimal couplings in SUGRA we include the gauge invariant function

$$
F_{\mathcal{R}}=\bar{\Phi} \Phi
$$

in the following $K$ 's

$K_{1 \mathcal{R}}=-N \ln \left(c_{\mathcal{R}}\left(F_{\mathcal{R}}+F_{\mathcal{R}}^{*}\right)-\frac{|\Phi|^{2}+|\bar{\Phi}|^{2}}{N}+F_{1 S}\right)$

which is completely logarithmic, and

$K_{2 \mathcal{R}}=-N \ln \left(c_{\mathcal{R}}\left(F_{\mathcal{R}}+F_{\mathcal{R}}^{*}\right)-\frac{|\Phi|^{2}+|\bar{\Phi}|^{2}}{N}\right)+F_{2 S}$,

which is polylogarithmic. In both cases we take $N>0$. The crucial difference of the $K$ 's considered here, compared to those employed in Refs. [30,31,84-87], is that unity does not accompany the terms $c_{\mathcal{R}}\left(F_{\mathcal{R}}+F_{\mathcal{R}}^{*}\right)$. As explained in Sect. 2.1.3, the identification of this quantity with unity at the vacuum of the theory essentially encapsulates the IG hypothesis - cf. Refs. [4,5,7-9]. The existence of the real function $|\Phi|^{2}+|\bar{\Phi}|^{2}$ inside the argument of logarithm is vital for this scenario, since otherwise the Kähler metric is singular. These terms provide canonical kinetic terms for $K=K_{1 \mathcal{R}}$ and $N=3$ in the Jordan frame or $c_{\mathcal{R}}$-dependent kinetic mixing in the remaining cases, as we show in the next section.

(b) $K$ 's with independent kinetic mixing. In this case we introduce a softly broken shift symmetry for the Higgs fields - cf. Refs. [32,56] - via the functions $F_{ \pm}=$ $\left|\Phi \pm \bar{\Phi}^{*}\right|^{2}$. In particular, the dominant shift symmetry adopted here is

$\Phi \rightarrow \Phi+c$ and $\bar{\Phi} \rightarrow \bar{\Phi}+c^{*}$ with $c \in \mathbb{C}$

under which $F_{-}$remains unaltered whereas $F_{+}$expresses the violation of this symmetry and is placed in the argument of a logarithm with coefficient $(-N)$, whereas $F_{-}$ is set outside it. Namely, we propose the following $K$ 's

$$
\begin{aligned}
& K_{1}=-N \ln \left(c_{+} F_{+}+F_{1 S}\left(|S|^{2}\right)\right)+c_{-} F_{-}, \\
& K_{2}=-N \ln \left(c_{+} F_{+}\right)+c_{-} F_{-}+F_{2 S}\left(|S|^{2}\right), \\
& K_{3}=-N \ln \left(c_{+} F_{+}\right)+F_{3 S}\left(F_{-},|S|^{2}\right),
\end{aligned}
$$

where $N>0$. As in the case of the $K$ 's in Eqs. (4a) and (4b) unity is not included in the argument of the logarithm. In the present case, the identification of $c_{+} F_{+}$ with unity - see Sect. 2.1.3 - at the vacuum of the theory incarnates the IG hypothesis - cf. Refs. [4,5,7-9]. The degree of the violation of the symmetry in Eq. (5) is expressed by $r_{ \pm}=c_{+} / c_{-}$, which is constrained by the unification condition to values of the order 0.1 - see Sect. 2.2.3. Since this value is quite natural we are not forced here to invoke any argument regarding its naturalness - cf. Ref. [35].

The models employing the $K$ 's in Eqs. (4a) and (4b) are more economical compared to the models based on the $K$ 's in Eqs. (6a)-(6c). Indeed, the latter include two parameters $\left(c_{+}\right.$and $\left.c_{-}\right)$from which one $\left(c_{+}\right)$enters $f_{\mathcal{R}}$ and the other $\left(c_{-}\right)$dominates independently the kinetic mixing - see below. However, these parameters are related to the shift symmetry in Eq. (5) which renders the relevant setting theoretically more appealing. Indeed, this symmetry has a string theoretical origin as shown in Refs. [88,89]. In this framework, mainly integer $N$ 's are considered which can be reconciled with the observational data - see Sect. 2.3.3. Namely, $N=3$ [N=2] for $K=K_{1}\left[K=K_{2}\right.$ or $\left.K_{3}\right]$ yields completely acceptable results. However, the deviation of the $N$ 's from these integer values is also acceptable [7-9,32,34,35,90,91] and assist us to cover the whole allowed domain of the observables.

Another possibility that could be inspected is what happens if we place the term $c_{-} F_{-}$inside the argument of the logarithm in Eqs. (6a) and (6b) - cf. Ref. [32] - considering the Kähler potentials

$$
\begin{aligned}
& K_{01}=-N \ln \left(c_{+} F_{+}-c_{-} F_{-} / N+F_{1 S}\right), \\
& K_{02}=-N \ln \left(c_{+} F_{+}-c_{-} F_{-} / N\right)+F_{2 S} .
\end{aligned}
$$

These $K$ 's, though, reduce to $K_{1 \mathcal{R}}$ and $K_{2} \mathcal{R}$ respectively if we set

$c_{+}=\frac{N c_{\mathcal{R}}-1}{2 N}$ and $c_{-}=\frac{N c_{\mathcal{R}}+1}{2}$.

For $c_{\mathcal{R}} \gg 1$ the arrangement above results in $r_{ \pm} \simeq 1 / N$. On the other hand, the same $r_{ \pm}$is found if we impose the unification constraint. Therefore, the observational predictions of the models based on the $K$ 's above are expected to be very similar to those obtained using Eqs. (4a) and (4b).

The functions $F_{l S}$ with $l=1,2,3$ encountered in Eqs. (4a), (4b) and (6a)-(6c) support canonical normalization and safe stabilization of $S$ during and after IGHI. Their possible forms are given in Ref. [35]. Just for definiteness, we adopt here only their logarithmic form, i.e.,

$$
\begin{aligned}
& F_{1 S}=-\ln \left(1+|S|^{2} / N\right), \\
& F_{2 S}=N_{S} \ln \left(1+|S|^{2} / N_{S}\right), \\
& F_{3 S}=N_{S} \ln \left(1+|S|^{2} / N_{S}+c_{-} F_{-} / N_{S}\right),
\end{aligned}
$$


with $0<N_{S}<6$. Recall [10-12,84-87] that the simplest term $|S|^{2}$ leads to instabilities for $K=K_{1}$ and light excitations for $K=K_{2}$ and $K_{3}$. The heaviness of these modes is required so that the observed curvature perturbation is generated wholly by our inflaton in accordance with the lack of any observational hint [97] for large non-Gaussianity in the cosmic microwave background.

\subsubsection{From Einstein to Jordan frame}

With the ingredients above we can extract the part of the Einstein frame (EF) action within SUGRA related to the complex scalars $z^{\alpha}=S, \Phi, \bar{\Phi}$ - denoted by the same superfield symbol. This has the form [84-87]

$\mathrm{S}=\int d^{4} x \sqrt{-\widehat{\mathfrak{g}}}\left(-\frac{1}{2} \widehat{\mathcal{R}}+K_{\alpha \bar{\beta}} \widehat{g}^{\mu \nu} D_{\mu} z^{\alpha} D_{\nu} z^{* \bar{\beta}}-\widehat{V}\right)$

where $\widehat{\mathcal{R}}$ is the EF Ricci scalar curvature, $D_{\mu}$ is the gauge covariant derivative, $K_{\alpha \bar{\beta}}=K_{, z^{\alpha} z^{* \bar{\beta}}}$, and $K^{\alpha \bar{\beta}} K_{\bar{\beta} \gamma}=\delta_{\gamma}^{\alpha}$. Also, $\widehat{V}$ is the EF SUGRA potential which can be found in terms of $W_{\mathrm{HI}}$ in Eq. (1) and the $K$ 's in Eqs. (6a)-(6c) via the formula

$\widehat{V}=e^{K}\left(K^{\alpha \bar{\beta}}\left(D_{\alpha} W_{\mathrm{HI}}\right) D_{\bar{\beta}}^{*} W_{\mathrm{HI}}^{*}-3\left|W_{\mathrm{HI}}\right|^{2}\right)+\frac{g^{2}}{2} \sum_{\mathrm{a}} \mathrm{D}_{\mathrm{a}}^{2}$,

where $D_{\alpha} W_{\mathrm{HI}}=W_{\mathrm{HI}, z^{\alpha}}+K_{, z^{\alpha}} W_{\mathrm{HI}}, \mathrm{D}_{\mathrm{a}}=z^{\alpha}\left(T_{\mathrm{a}}\right)_{\alpha}^{\beta} K_{\beta}$ and the summation is applied over the generators $T_{\mathrm{a}}$ of $G_{B-L}$. In the right-hand side (r.h.s) of the equation above we clearly recognize the contribution from the $\mathrm{D}$ terms (proportional to $g^{2}$ ) and the remaining one which comes from the F terms.

If we perform a conformal transformation, along the lines of Refs. [32,84-87], defining the frame function as

$-\Omega / N=\exp (-K / N) \Rightarrow K=-N \ln (-\Omega / N)$,

we can obtain the form of S in the Jordan Frame ( JF) which is written as [32]

$$
\begin{aligned}
\mathrm{S}= & \int d^{4} x \sqrt{-\mathfrak{g}}\left(\frac{\Omega}{2 N} \mathcal{R}-\frac{27}{N^{3}} \Omega \mathcal{A}_{\mu} \mathcal{A}^{\mu}-V\right. \\
& \left.+\left(\Omega_{\alpha \bar{\beta}}+\frac{3-N}{N} \frac{\Omega_{\alpha} \Omega_{\bar{\beta}}}{\Omega}\right) D_{\mu} z^{\alpha} D^{\mu} z^{* \bar{\beta}}\right),
\end{aligned}
$$

where we use the shorthand notation $\Omega_{\alpha}=\Omega_{, z^{\alpha}}$, and $\Omega_{\bar{\alpha}}=$ $\Omega_{, z^{* \bar{\alpha}}}$. We also set $V=\widehat{V} \Omega^{2} / N^{2}$ and

$$
\mathcal{A}_{\mu}=-i N\left(\Omega_{\alpha} D_{\mu} z^{\alpha}-\Omega_{\bar{\alpha}} D_{\mu} z^{* \bar{\alpha}}\right) / 6 \Omega \text {. }
$$

Computing the expression in the parenthesis of the second line in Eq. (12a) for $K=K_{1 \mathcal{R}}$ and $K_{2 \mathcal{R}}$, we can easily verify that the choice for $N=3$ ensures canonical kinetic terms - in accordance with the findings in Refs. [30,31,8487] - whereas in the remaining cases a $c_{\mathcal{R}^{-}}$(and not $\phi$-) dependent kinetic mixing emerges. Indeed, in any case we have $\Omega_{\alpha \bar{\beta}}=\delta_{\alpha \bar{\beta}}$ and for $N=3$ the second term in the parenthesis vanishes. On the contrary, for $K=K_{1}, K_{2}$ and $K_{3}$, the same expression is not only different than $\delta_{\alpha \bar{\beta}}$ but also includes ( $\phi$-dependent) entries proportional to and dominated by $c_{-} \gg c_{+}$. For this reason, the relevant models of IGHI may be more properly characterized as kinetically modified. The non-renormalizability of this kinetic mixing is under control since $\phi \ll 1$ and the theory is trustable up to $m_{\mathrm{P}}$, as we show in Sect. 2.3.2.

Most importantly, though, the first term in the first line of the r.h.s of Eq. (12a) reveals that $-\Omega / N$ plays the role of a non-minimal coupling to gravity. Comparing Eq. (11) with the $K$ 's in Eqs. (4a)-(6c) we can infer that

$-\frac{\Omega}{N}= \begin{cases}2\left(N c_{\mathcal{R}}+1\right) F_{\mathcal{R}} / N & \text { for } K=K_{1 \mathcal{R}} \text { and } K_{2 \mathcal{R}}, \\ c_{+} F_{+} & \text {for } K=K_{1}, K_{2} \text { and } K_{3},\end{cases}$

along the field configuration

$\Phi=\bar{\Phi}^{*}$ and $S=0$

which is a honest inflationary trajectory, as shown in Sect. 2.2.2. The identification of the quantity in Eq. (13) with $m_{\mathrm{P}}^{2}$ at the vacuum, according to the IG conjecture, can be accommodated as described in the next section.

\subsubsection{Induced-gravity requirement}

The implementation of the IG scenario requires the generation of $m_{\mathrm{P}}$ at the vacuum of the theory, which thereby has to be determined. To do this we have to compute $V$ in Eq. (10b) for small values of the various fields, expanding it in powers of $1 / m_{\mathrm{P}}$. Namely, we obtain the following low-energy effective potential

$V_{\mathrm{eff}}=e^{\widetilde{K}} \widetilde{K}^{\alpha \bar{\beta}} W_{\mathrm{HI} \alpha} W_{\mathrm{HI} \bar{\beta}}^{*}+\frac{g^{2}}{2} \sum_{\mathrm{a}} \mathrm{D}_{\mathrm{a}}^{2}+\cdots$,

where the ellipsis represents terms proportional to $W_{\mathrm{HI}}$ or $\left|W_{\mathrm{HI}}\right|^{2}$ which obviously vanish along the path in Eq. (68) - we assume here that the vacuum is contained in the inflarionary trajectory. Also, $\widetilde{K}$ is the limit of the $K$ 's in Eqs. (4a)-(6c) for $m_{\mathrm{P}} \rightarrow \infty$. The absence of unity in the arguments of the logarithms multiplied by $N$ in these $K$ 's prevents the drastic simplification of $\widetilde{K}$, especially for $K=K_{1 \mathcal{R}}$ and $K_{1}-$ cf. Ref. [35]. As a consequence, the expression of the 
resulting $V_{\text {eff }}$ is rather lengthy. For this reason we confine ourselves below to $K=K_{2}$ or $K_{3}$ where $F_{l S}$ with $l=$ 2, 3 is placed outside the first logarithm and so $\widetilde{K}$ can be significantly simplified. Namely, we get

$\widetilde{K}=-N \ln c_{+} F_{+}+c_{-} F_{-}+|S|^{2}$,

from which we can then compute

$$
\left(\widetilde{K}_{\alpha \bar{\beta}}\right)=\operatorname{diag}\left(\widetilde{M}_{ \pm}, 1\right) \text { with } \quad \widetilde{M}_{ \pm}=\left(\begin{array}{ll}
c_{\widetilde{K}} & \widetilde{K}_{\Phi \bar{\Phi}^{*}} \\
\widetilde{K}_{\Phi \bar{\Phi}^{*}} & c_{-}
\end{array}\right)
$$

Here,

$\widetilde{K}_{\Phi \bar{\Phi}^{*}}=\frac{N}{\left(\Phi+\bar{\Phi}^{*}\right)^{2}} \quad$ and $\quad \widetilde{K}_{\bar{\Phi} \Phi^{*}}=\frac{N}{\left(\Phi^{*}+\bar{\Phi}\right)^{2}}$

since

$\widetilde{K}_{\Phi}=-N /\left(\Phi+\bar{\Phi}^{*}\right)+c_{-}\left(\Phi^{*}-\bar{\Phi}\right)$

and

$\widetilde{K}_{\bar{\Phi}}=-N /\left(\Phi^{*}+\bar{\Phi}\right)-c_{-}\left(\Phi-\bar{\Phi}^{*}\right)$.

To compute $V_{\text {eff }}$ we need to know

$$
\left(\widetilde{K}^{\alpha \bar{\beta}}\right)=\operatorname{diag}\left(\tilde{M}_{ \pm}^{-1}, 1\right)
$$

where

$\widetilde{M}_{ \pm}^{-1}=\frac{1}{\operatorname{det} \widetilde{M}_{ \pm}}\left(\begin{array}{cc}c_{-} & -\widetilde{K}_{\Phi \bar{\Phi}^{*}} \\ -\widetilde{K}_{\bar{\Phi} \Phi^{*}} & c_{-}\end{array}\right)$

with

$\operatorname{det} \tilde{M}_{ \pm}=c_{-}^{2}-N^{2} / F_{+}^{2}$.

Upon substitution of Eqs. (17a) and (17b) into Eq. (15a) we obtain

$$
\begin{aligned}
& V_{\mathrm{eff}} \simeq \lambda^{2} e^{\widetilde{K}_{+}}\left|\bar{\Phi} \Phi-\frac{1}{4} M^{2}\right|^{2}+\frac{g^{2}}{2}\left(\Phi \widetilde{K}_{\Phi}-\bar{\Phi} \widetilde{K}_{\bar{\Phi}}\right)^{2} \\
& +\frac{\lambda^{2} e^{\widetilde{K}_{+}}|S|^{2}}{\operatorname{det} \widetilde{M}_{ \pm}}\left(c_{-}\left(|\Phi|^{2}+|\bar{\Phi}|^{2}\right)-\widetilde{K}_{\Phi \bar{\Phi}^{*}} \bar{\Phi}^{*} \Phi-\widetilde{K}_{\bar{\Phi} \Phi^{*}} \bar{\Phi} \Phi^{*}\right)
\end{aligned}
$$

where $\widetilde{K}_{+}=-N \ln c_{+} F_{+}$. We remark that the direction in Eq. (68) assures D-flatness since $\left\langle\Phi \widetilde{K}_{\Phi}\right\rangle=\left\langle\bar{\Phi} \widetilde{K}_{\bar{\Phi}}\right\rangle$ and so the vacuum lies along it with

$$
\langle S\rangle=0 \text { and }|\langle\Phi\rangle|=|\langle\bar{\Phi}\rangle|=M / 2
$$

The same result holds also for $K=K_{1 \mathcal{R}}, K_{2 \mathcal{R}}$ and $K_{1}$ as we can verify after a more tedious computation. Equation (19) means that $\langle\Phi\rangle$ and $\langle\bar{\Phi}\rangle$ spontaneously break $U(1)_{B-L}$ down to $\mathbb{Z}_{2}^{B-L}$. Note that $U(1)_{B-L}$ is already broken during IGHI and so no cosmic string are formed - contrary to what happens in the models of the standard F-term hybrid inflation [37-42], which also employ $W_{\mathrm{HI}}$ in Eq. (1).

Inserting Eqs. (19) into (13) we deduce that the conventional Einstein gravity can be recovered at the vacuum if

$$
M= \begin{cases}\sqrt{2 N /\left(N c_{\mathcal{R}}-1\right)} & \text { for } K=K_{1 \mathcal{R}} \text { and } K_{2 \mathcal{R}}, \\ 1 / \sqrt{c_{+}} & \text {for } K=K_{1}, K_{2} \text { and } K_{3} .\end{cases}
$$

For $c_{\mathcal{R}} \simeq 10^{4}$ or $c_{+} \sim\left(10^{2}-10^{3}\right)$ employed here, the resulting values of $M$ are theoretically quite natural since they lie close to unity. Indeed, since the form of $W_{\mathrm{HI}}$ in Eq. (1) is established around $m_{\mathrm{P}}$ we expect that the scales entered by hand in the theory have comparable size.

\subsection{Inflationary potential}

Below we outline the derivation of the inflationary potential in Sect. 2.2.1 and check its stability by computing one-loop corrections in Sect. 2.2.2. The last part of the analysis allows us to determine the gauge-coupling unification condition (see Sect. 2.2.3) which assists us to further constrain our models.

\subsubsection{Tree-level result}

If we express $\Phi, \bar{\Phi}$ and $S$ according to the parametrization

$\Phi=\frac{\phi e^{i \theta}}{\sqrt{2}} \cos \theta_{\Phi}, \quad \bar{\Phi}=\frac{\phi e^{i \bar{\theta}}}{\sqrt{2}} \sin \theta_{\Phi} \quad$ and $\quad S=\frac{s+i \bar{s}}{\sqrt{2}}$

with $0 \leq \theta_{\Phi} \leq \pi / 2$, the trough in Eq. (68) can be written as

$\bar{s}=s=\theta=\bar{\theta}=0$ and $\theta_{\Phi}=\pi / 4$.

Along this the only surviving term in Eq. (10b) is

$\widehat{V}_{\mathrm{HI}}=e^{K} K^{S S^{*}}\left|W_{\mathrm{HI}, S}\right|^{2}$,

which, for the choices of $K$ 's in Eqs. (6a)-(6c), reads

$$
\widehat{V}_{\mathrm{HI}}=\frac{\lambda^{2} f_{\mathrm{W}}^{2}}{16 \mathrm{a}_{W}^{2}} f_{\mathcal{R}}^{-N} \cdot \begin{cases}f_{\mathcal{R}} & \text { for } K=K_{1 \mathcal{R}}, K_{1}, \\ 1 & \text { for } K=K_{2 \mathcal{R}}, K_{2} \text { and } K_{3},\end{cases}
$$


where $f_{\mathcal{R}}^{-N}=e^{K}$ and we define the (inflationary) frame function as

$f_{\mathcal{R}}=-\left.\frac{\Omega}{N}\right|_{\text {Eq. (22) }}$

which is translated as

$$
f_{\mathcal{R}}= \begin{cases}\left(N c_{\mathcal{R}}-1\right) \phi^{2} / 2 N & \text { for } K=K_{1 \mathcal{R}} \text { and } K_{2 \mathcal{R}} \\ c_{+} \phi^{2} & \text { for } K=K_{1}, K_{2} \text { and } K_{3}\end{cases}
$$

The last factor in Eq. (23b) originates from the expression of $K^{S S^{*}}$ for the various $K$ 's. Also

$$
f_{\mathrm{W}}= \begin{cases}\left(N c_{\mathcal{R}}-1\right) \phi^{2}-2 N & \text { for } K=K_{1 \mathcal{R}} \text { and } K_{2 \mathcal{R}}, \\ c_{+} \phi^{2}-1 & \text { for } K=K_{1}, K_{2} \text { and } K_{3}\end{cases}
$$

arises from the last factor in the r.h.s of Eq. (23a) together with $\mathrm{a}_{W}=\left(N c_{\mathcal{R}}-1\right)$ for $K=K_{1 \mathcal{R}}$ and $K_{2 \mathcal{R}}$ and $\mathrm{a}_{W}=c_{+}$ for $K=K_{1}, K_{2}$ and $K_{3}$. If we set

$N= \begin{cases}2 n+3 & \text { for } K=K_{1 \mathcal{R}}, K_{1}, \\ 2(n+1) & \text { for } K=K_{2 \mathcal{R}}, K_{2} \text { and } K_{3},\end{cases}$

we arrive at a universal expression for $\widehat{V}_{\mathrm{HI}}$ which is

$\widehat{V}_{\mathrm{HI}}=\frac{\lambda^{2} f_{\mathrm{W}}^{2}}{16 \mathrm{a}_{W}^{2} f_{\mathcal{R}}^{2(1+n)}}$

The value $n=0$ is special since we get $N=3$ for $K=$ $K_{1 \mathcal{R}}$ and $K_{1}$ or $N=2$ for $K=K_{2 \mathcal{R}}, K_{2}$ or $K_{3}$. Therefore, $\widehat{V}_{\mathrm{HI}}$ develops an inflationary plateau as in the original case of Starobinsky model within no-scale SUGRA [4,5,10-12] for large $c_{\mathcal{R}}$ or $c_{+}$. Contrary to that case, though, here we also have $n$ and $c_{-}$, whose variation may have an important impact on the observables - cf. Refs. [32,34]. In particular, for $n<0, \widehat{V}_{\mathrm{HI}}$ remains an increasing function of $\phi$, whereas for $n>0$, it develops a local maximum

$\widehat{V}_{\mathrm{HI}}\left(\phi_{\max }\right)=\frac{\lambda^{2} n^{2 n}}{16 \mathrm{a}^{2}(1+n)^{2(1+n)}}$ at $\phi_{\max }=\sqrt{\frac{1+n}{\mathrm{a} n}}$,

where a $=c_{\mathcal{R}} / 2$ for $K=K_{1 \mathcal{R}}$ and $K_{2 \mathcal{R}}$ whereas a $=c_{+}$ for $K=K_{1}, K_{2}$ and $K_{3}$. In a such case we are forced to assume that hilltop [92] IGHI occurs with $\phi$ rolling from the region of the maximum down to smaller values. The relevant tuning of the initial conditions can be quantified by defining [38-42] the quantity

$\Delta_{\max }=\left(\phi_{\max }-\phi_{\star}\right) / \phi_{\max }$, where $\phi_{\star}$ is the value of $\phi$ when the pivot scale $k_{\star}=$ $0.05 / \mathrm{Mpc}$ crosses outside the inflationary horizon. The naturalness of the attainment of IGHI increases with $\Delta_{\max \star}$, and it is maximized when $\phi_{\max } \gg \phi_{\star}$ which results in $\Delta_{\max \star} \simeq 1$.

To specify the EF canonically normalized inflaton, we note that, for all choices of $K$ in Eqs. (4a), (4b) and (6a)-(6c), $K_{\alpha \bar{\beta}}$ along the configuration in Eq. (22) takes the form

$$
\left(K_{\alpha \bar{\beta}}\right)=\operatorname{diag}\left(M_{ \pm}, K_{S S^{*}}\right)
$$

where $K_{S S^{*}}=1 / f_{\mathcal{R}}\left[K_{S S^{*}}=1\right]$ for $K=K_{1 \mathcal{R}}, K_{1}[K=$ $K_{2 \mathcal{R}}, K_{2}$ and $\left.K_{3}\right]$. For $K=K_{1 \mathcal{R}}$ and $K_{2 \mathcal{R}}$ we find

$M_{ \pm}=\left(\begin{array}{cc}\left(1+N c_{\mathcal{R}}\right) / 2 f_{\mathcal{R}} & N / \phi^{2} \\ N / \phi^{2} & \left(1+N c_{\mathcal{R}}\right) / 2 f_{\mathcal{R}}\end{array}\right)$.

and upon diagonalization we obtain the following eigenvalues

$\kappa_{+}=N c_{\mathcal{R}} f_{\mathcal{R}}^{-1}$ and $\quad \kappa_{-}=f_{\mathcal{R}}^{-1}$.

Note that the existence of the real function $|\Phi|^{2}+|\bar{\Phi}|^{2}$ inside the argument of logarithm is vital for this scenario, since otherwise $M_{ \pm}$develops zero eigenvalue and so it is singular, i.e., no $K^{\alpha \bar{\beta}}$ can be defined. On the other hand, for $K=$ $K_{1}, K_{2}$ and $K_{3}$ we obtain

$M_{ \pm}=\left(\begin{array}{cc}c_{-} & N / \phi^{2} \\ N / \phi^{2} & c_{-}\end{array}\right)$

with eigenvalues

$\kappa_{ \pm}=c_{-} \pm N / \phi^{2}$

Given that the lowest $\phi$ value is given in Eq. (20), we can impose, in this case, a robust restriction on the parameters to assure the positivity of $\kappa_{-}$during and after IGHI. Namely,

$\kappa_{-} \gtrsim 0 \Rightarrow r_{ \pm} \lesssim 1 / N$

whereas we are not obliged to impose any condition for $K=$ $K_{1 \mathcal{R}}$ and $K_{2 \mathcal{R}}$.

Inserting Eqs. (21) and (30) in the second term of the r.h.s of Eq. (10a) we can define the EF canonically normalized fields, denoted by hat, as follows

$$
\begin{aligned}
& \frac{d \widehat{\phi}}{d \phi}=J=\sqrt{\kappa_{+}}, \widehat{\theta}_{+}=\frac{J \phi \theta_{+}}{\sqrt{2}}, \widehat{\theta}_{-}=\sqrt{\frac{\kappa_{-}}{2}} \phi \theta_{-}, \\
& \widehat{\theta}_{\Phi}=\phi \sqrt{\kappa_{-}}\left(\theta_{\Phi}-\pi / 4\right), \quad(\widehat{s}, \widehat{\bar{s}})=\sqrt{K_{S S^{*}}}(s, \bar{s}),
\end{aligned}
$$

where $\theta_{ \pm}=(\bar{\theta} \pm \theta) / \sqrt{2}$. Note, in passing, that the spinors $\psi_{S}$ and $\psi_{\Phi \pm}$ associated with the superfields $S$ and $\Phi-\bar{\Phi}$ are similarly normalized, i.e., $\widehat{\psi}_{S}=\sqrt{K_{S S^{*}}} \psi_{S}$ and $\widehat{\psi}_{\Phi \pm}=$ $\sqrt{\kappa_{ \pm}} \psi_{\Phi \pm}$ with $\psi_{\Phi \pm}=\left(\psi_{\Phi} \pm \psi_{\bar{\Phi}}\right) / \sqrt{2}$. 


\subsubsection{Stability and loop-corrections}

We can verify that the inflationary direction in Eq. (22) is stable w.r.t the fluctuations of the non-inflaton fields. To this end, we construct the mass-squared spectrum of the various scalars defined in Eqs. (33a) and (33b). Taking the limit $c_{-} \gg$ $c_{+}$we find the expressions of the masses squared $\widehat{m}_{\chi^{\alpha}}^{2}$ (with $\chi^{\alpha}=\theta_{+}, \theta_{\Phi}$ and $S$ ) arranged in Table 1. For $\phi \simeq \phi_{\star}$ these fairly approach the quite lengthy, exact expressions taken into account in our numerical computation. Given that $\phi<0.1$ for $K=K_{1 \mathcal{R}}$ and $f_{\mathrm{W}} \gg 1$ for $K=K_{1}$ we deduce that $\widehat{m}_{s}^{2}>0$ for $N \simeq 3$. Also for $K=K_{2 \mathcal{R}}, K_{2}$ or $K_{3}$ and $0<N_{S}<6, \widehat{m}_{s}^{2}>0$ stays positive and heavy enough, i.e. $\widehat{m}_{z^{\alpha}}^{2} \gg \widehat{H}_{\mathrm{HI}}^{2}=\widehat{V}_{\mathrm{HI}} / 3$. In Table 1 we also display the masses, $M_{B L}$, of the gauge boson $A_{B L}$ - which signals the fact that $G_{B-L}$ is broken during IGHI - and the masses of the corresponding fermions. Note that the unspecified eigestate $\widehat{\psi}_{ \pm}$is defined as

$\widehat{\psi}_{ \pm}=\left(\widehat{\psi}_{\Phi+} \pm \widehat{\psi}_{S}\right) / \sqrt{2}$.

As a consequence, let us again emphasize that no cosmic string are produced at the end of IGHI.

The derived mass spectrum can be employed in order to find the one-loop radiative corrections, $\Delta \widehat{V}_{\mathrm{HI}}$, to $\widehat{V}_{\mathrm{HI}}$. Considering SUGRA as an effective theory with cutoff scale equal to $m_{\mathrm{P}}$, the well-known Coleman-Weinberg formula [93] can be employed taking into account only the masses which lie well below $m_{\mathrm{P}}$, i.e., all the masses arranged in Table 1 besides $M_{B L}$ and $\widehat{m}_{\theta_{\Phi}}$ - note that these contributions are cancelled out for $K=K_{1 \mathcal{R}}$ and $N=3$ or $K=K_{2 \mathcal{R}}$ and $N=2$. The resulting $\Delta \widehat{V}_{\mathrm{HI}}$ leaves intact our inflationary outputs, provided that the renormalization-group mass scale $\Lambda$, is determined by requiring $\Delta \widehat{V}_{\mathrm{HI}}\left(\phi_{\star}\right)=0$ or $\Delta \widehat{V}_{\mathrm{HI}}\left(\phi_{\mathrm{f}}\right)=0$. These conditions yield $\Lambda \simeq 3.2 \cdot 10^{-5}-1.4 \cdot 10^{-4}$ and render our results practically independent of $\Lambda$ since these can be derived exclusively by using $\widehat{V}_{\mathrm{HI}}$ in Eq. (24) with the various quantities evaluated at $\Lambda$ - cf. Ref. [32]. Note that their renormalization-group running is expected to be negligible because $\Lambda$ is close to the inflationary scale $\widehat{V}_{\mathrm{HI}}^{1 / 4} \simeq$ $(3-7) \cdot 10^{-3}$. Recall, here, that in the case of F-term hybrid inflation [36-45] the SUSY potential is classically flat and the radiative corrections contribute (together with the SUGRA corrections) in the inclination of the inflationary path.

\subsubsection{SUSY gauge coupling unification}

The mass $M_{B L}$ listed in Table 1 of the gauge boson $A_{B L}$ may, in principle, be a free parameter since the $U(1)_{B-L}$ gauge symmetry does not disturb the unification of the MSSM gauge coupling constants. To be more specific, though, we prefer to determine $M_{B L}$ by requiring that it takes the value $M_{\mathrm{GUT}}$ dictated by this unification at the vacuum of the theory.

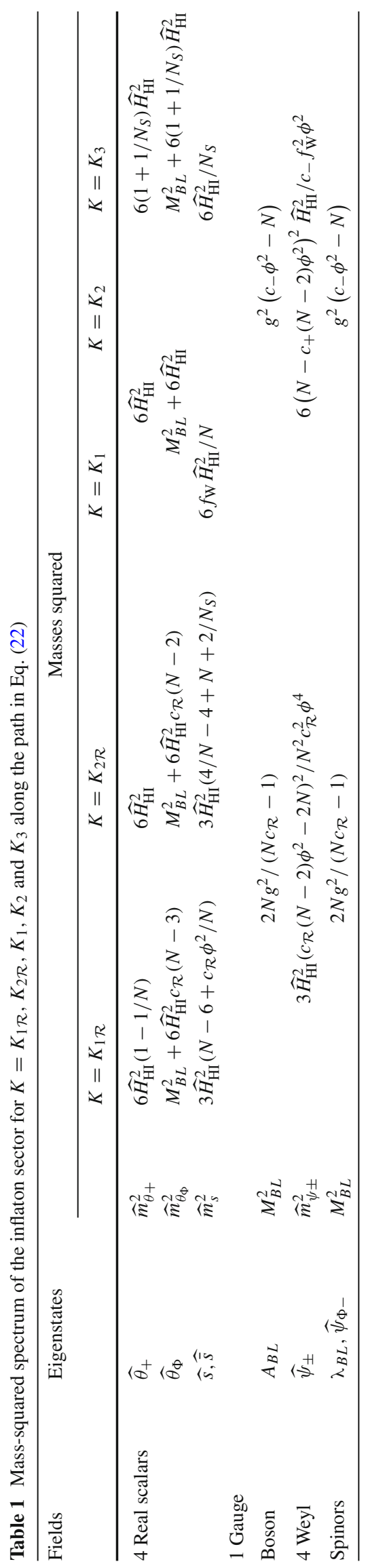


Namely, we impose

$$
\left\langle M_{B L}\right\rangle=M_{\mathrm{GUT}} \simeq 2 / 2.43 \cdot 10^{-2}=8.22 \cdot 10^{-3} .
$$

This simple principle has important consequences for both classes of models considered here. In particular:

(a) For $K=K_{1 \mathcal{R}}$ or $K_{2 \mathcal{R}}$. In this cases, the condition above completely determines $c_{\mathcal{R}}$ since it implies via the findings of Table 1

$c_{\mathcal{R}}=\frac{1}{N}+\frac{2 g^{2}}{M_{\mathrm{GUT}}^{2}} \simeq 1.451 \cdot 10^{4}$,

leading to $M \simeq 0.0117$ via Eq. (20). Here we take $g \simeq$ 0.7 which is the value of the unified coupling constant within MSSM. Although $c_{\mathcal{R}}$ above is very large, there is no problem with the validity of the effective theory, in accordance with the results of earlier works $[4,5,7-$ 9,20 ] on IG inflation with gauge singlet inflaton. Indeed, expanding about $\langle\phi\rangle=M$ - see Eq. (20) - the second term in the r.h.s of Eq. (10a) for $\mu=v=0$ and $\widehat{V}_{\mathrm{HI}}$ in Eq. (24) we obtain

$J^{2} \dot{\phi}^{2} \simeq\left(1-\sqrt{\frac{2}{N}} \widehat{\delta \phi}+\frac{3}{2 N} \widehat{\delta \phi}^{2}-\sqrt{\frac{2}{N^{3}}} \widehat{\delta \phi}^{3}+\cdots\right) \widehat{\delta \phi}^{2}$,

where $\widehat{\delta \phi}$ is the canonically normalized inflaton at the vacuum - see Sect. IIIC1 - and

$\widehat{V}_{\mathrm{HI}} \simeq \frac{\lambda^{2} \widehat{\delta \phi}^{2}}{2 N c_{\mathcal{R}}^{2}}\left(1-\frac{2 N-1}{\sqrt{2 N}} \widehat{\delta \phi}+\frac{8 N^{2}-4 N+1}{8 N} \widehat{\delta \phi}^{2}+\cdots\right)$.

These expressions indicate that $\Lambda_{\mathrm{UV}}=m_{\mathrm{P}}$, since $c_{\mathcal{R}}$ does not appear in any of the numerators above. Although these expansions are valid only during reheating we consider $\Lambda_{\mathrm{UV}}$ extracted this way as the overall cut-off scale of the theory since reheating is regarded [26] as an unavoidable stage of IGHI.

(b) For $K=K_{1}, K_{2}$ or $K_{3}$. In this cases, the condition above allows us to fix $r_{ \pm}$since, substituting Eq. (20) in $M_{B L}$ shown in Table 1, we obtain

$$
g^{2}\left(c_{-}\langle\phi\rangle^{2}-N\right)=M_{\mathrm{GUT}}^{2} \Rightarrow r_{ \pm}=\frac{g^{2}}{N g^{2}+M_{\mathrm{GUT}}^{2}} .
$$

Since $M_{\mathrm{GUT}}>0$ the condition above satisfies the restriction in Eq. (32) yielding $r_{ \pm}$close to its upper bound because $M_{\text {GUT }} \ll 1$.
As a bottom line, under the assumption in Eq. (35), $c_{\mathcal{R}}$ for $K=K_{1 \mathcal{R}}$ and $K_{2 \mathcal{R}}$ or $r_{ \pm}$for $K=K_{1}, K_{2}$ and $K_{3}$ cease to be free parameters, in sharp contrast to the models of Refs. [30-35] where the same assumption is employed to extract $M \ll 1$ as a function of the free parameters without any other theoretical constraint between them. Therefore, the interplay of Eqs. (20) and (38) leads to the reduction of the free parameters by one, thereby rendering the present set-up more restrictive and predictive.

\subsection{Inflation analysis}

In Sects. 2.3.2 and 2.3.3 below we inspect analytically and numerically respectively, if the potential in Eq. (24) endowed with the condition of Eqs. (20) and (38) may be consistent with a number of observational constraints introduced in Sect. 2.3.1.

\subsubsection{General framework}

Given that the analysis of inflation in both EF and JF yields equivalent results $[17,18]$, we carry it out exclusively in the EF. In particular, the period of slow-roll IGHI is determined in the EF by the condition

$\max \{\widehat{\epsilon}(\phi),|\widehat{\eta}(\phi)|\} \leq 1$,

where the slow-roll parameters $[94,95]$,

$\widehat{\epsilon}=\left(\widehat{V}_{\mathrm{HI}, \widehat{\phi}} / \sqrt{2} \widehat{V}_{\mathrm{HI}}\right)^{2}$ and $\hat{\eta}=\widehat{V}_{\mathrm{HI}, \widehat{\phi} \hat{\phi}} / \widehat{V}_{\mathrm{HI}} \cdot$

The number of e-foldings $\widehat{N}_{\star}$ that the scale $k_{\star}=0.05 / \mathrm{Mpc}$ experiences during IGHI and the amplitude $A_{\mathrm{S}}$ of the power spectrum of the curvature perturbations generated by $\phi$ can be computed using the standard formulae $[94,95]$

$\widehat{N}_{\star}=\int_{\widehat{\phi}_{\mathrm{f}}}^{\widehat{\phi}_{\star}} d \widehat{\phi} \frac{\widehat{V}_{\mathrm{HI}}}{\widehat{V}_{\mathrm{HI}, \widehat{\phi}}}$ and $A_{\mathrm{s}}^{1 / 2}=\frac{1}{2 \sqrt{3} \pi} \frac{\widehat{V}_{\mathrm{HI}}^{3 / 2}\left(\widehat{\phi}_{\star}\right)}{\left|\widehat{V}_{\mathrm{HI}, \widehat{\phi}}\left(\widehat{\phi}_{\star}\right)\right|}$,

where $\phi_{\star}\left[\widehat{\phi}_{\star}\right]$ is the value of $\phi[\widehat{\phi}]$ when $k_{\star}$ crosses the inflationary horizon. These observables are to be confronted with the requirements [97]

$$
\begin{aligned}
\widehat{N}_{\star} & \simeq 61.5+\ln \frac{\widehat{V}_{\mathrm{HI}}\left(\phi_{\star}\right)^{1 / 2}}{\widehat{V}_{\mathrm{HI}}\left(\phi_{\mathrm{f}}\right)^{1 / 4}}+\frac{1}{2} f_{\mathcal{R}}\left(\phi_{\star}\right) ; \\
A_{\mathrm{s}}^{1 / 2} & \simeq 4.627 \cdot 10^{-5} .
\end{aligned}
$$

Note that in Eq. (41a) we consider an equation-of-state parameter $w_{\text {int }}=1 / 3$ corresponding to quartic potential which is expected to approximate $\widehat{V}_{\mathrm{HI}}$ rather well for $\phi \ll 1$ - see Ref. [32]. We obtain $\widehat{N}_{\star} \simeq(57.5-60)$. 
Then, we compute the remaining inflationary observables, i.e., the (scalar) spectral index $n_{\mathrm{s}}$, its running $a_{\mathrm{s}}$, and the scalar-to-tensor ratio $r$ which are found from the relations $[94,95]$

$n_{\mathrm{s}}=1-6 \widehat{\epsilon}_{\star}+2 \widehat{\eta}_{\star}, \quad r=16 \widehat{\epsilon}_{\star}$,

$a_{\mathrm{s}}=2\left(4 \widehat{\eta}_{\star}^{2}-\left(n_{\mathrm{s}}-1\right)^{2}\right) / 3-2 \widehat{\xi}_{\star}$,

where the variables with subscript $\star$ are evaluated at $\phi=\phi_{\star}$ and $\widehat{\xi}=\widehat{V}_{\mathrm{HI}, \widehat{\phi}} \widehat{V}_{\mathrm{HI}, \widehat{\phi} \widehat{\phi} \widehat{\phi}} / \widehat{V}_{\mathrm{HI}}^{2}$.

The resulting values of $n_{\mathrm{s}}$ and $r$ must be in agreement with the fitting of the data $[23,59]$ with $\Lambda \mathrm{CDM}+r$ model. We take into account the data from Planck and Baryon Acoustic Oscillations (BAO) and the BK14 data taken by the BICEP2/Keck Array CMB polarization experiments up to and including the 2014 observing season. The results are
(a) $n_{\mathrm{s}}=0.968 \pm 0.009$ and
(b) $r \leq 0.07$,

at $95 \%$ confidence level (c.I.) with $\left|a_{\mathrm{s}}\right| \ll 0.01$.

\subsubsection{Analytic results}

A crucial difference of the present analysis w.r.t that for the models in Refs. [30-35] is that $M$, given by Eq. (20), is not negligible during the inflationary period and enters the relevant formulas via the function $f_{\mathrm{W}}$ defined below Eq. (23b). We find it convenient to expose separately our results for the two basic classes of models introduced in Sect. 2.1.1. Namely:

(a) For $K=K_{1 \mathcal{R}}$ and $K_{2 \mathcal{R}}$. The slow-roll parameters can be derived employing $J$ in Eq. (24), without explicitly expressing $\widehat{V}_{\mathrm{HI}}$ in terms of $\widehat{\phi}$. Our results are

$\widehat{\epsilon}=4 \frac{\tilde{f}_{\mathrm{W}}^{2}\left(n \tilde{f}_{\mathrm{W}}-2\right)^{2}}{N c_{\mathcal{R}}^{4} \phi^{8}}$ and $\hat{\eta}=8 \frac{2-\tilde{f}_{\mathrm{W}}-4 n \tilde{f}_{\mathrm{W}}+n^{2} \tilde{f}_{\mathrm{W}}^{2}}{N \tilde{f}_{\mathrm{W}}^{2}}$,

where $\tilde{f}_{\mathrm{W}}=c_{\mathcal{R}} \phi^{2}-2$. The condition Eq. (39a) is violated for $\phi=\phi_{\mathrm{f}}$, which is found to be

$\phi_{\mathrm{f}} \simeq \max \left(\frac{2}{\sqrt{c_{\mathcal{R}}}} \sqrt{\frac{1+n}{2 n+\sqrt{N}}}, 2 \sqrt{\frac{2}{c_{\mathcal{R}}}} \sqrt{\frac{1-4 n}{8 n^{2}+N}}\right)$.

Then, $\widehat{N}_{\star}$ can be also computed from Eq. (40) as follows

$$
\widehat{N}_{\star} \simeq \begin{cases}N c_{\mathcal{R}} \phi_{\star}^{2} / 8 & \text { for } n=0, \\ N \ln \left(\frac{2(1+n)}{2-n \tilde{f}_{W *}}\right) / 4 n(1+n) & \text { for } n \neq 0,\end{cases}
$$

where $\tilde{f}_{W *}=\tilde{f}_{\mathrm{W}}\left(\widehat{\phi}_{\star}\right)$. Solving the above equations w.r.t $\phi_{\star}$ we obtain a unified expression

$\phi_{\star} \simeq \sqrt{\frac{2 f_{\mathcal{R}_{\star}}}{c_{\mathcal{R}}}}$ with $f_{\mathcal{R} \star}=\frac{1+n}{n}\left(1-e^{-4 n(1+n) \widehat{N}_{\star} / N}\right)$

reducing to $4 \widehat{N}_{\star} / N$ in the limit $n \rightarrow 0$. For $c_{\mathcal{R}}$ in Eq. (36) we can verify that $\phi_{\star} \sim 0.1$ and so the model is (automatically) well stabilized against corrections from higher order terms of the form $(\Phi \bar{\Phi})^{p}$ with $p>1$ in $W_{\mathrm{HI}}-$ see Eq. (1). Thanks to Eq. (36), we can derive uniquely $\lambda$ from the expression

$\lambda=8 \sqrt{6 A_{\mathrm{s}}} \pi c_{\mathcal{R}} f_{\mathcal{R} \star}^{n+1} \frac{n\left(1-f_{\mathcal{R}_{\star}}\right)+1}{\sqrt{N}\left(f_{\mathcal{R}_{\star}}-1\right)^{2}}$,

applying the second equation in Eq. (40). Upon substitution of $f_{\mathcal{R} \star}$ into Eq. (42a) we obtain the predictions of the model which are

$$
\begin{aligned}
n_{\mathrm{S}} & \simeq 1-\frac{8 n^{2}}{N}+\frac{16}{N} \frac{n}{f_{\mathcal{R}_{\star}}-1}-\frac{8}{N} \frac{f_{\mathcal{R}_{\star}}+1}{\left(f_{\mathcal{R}_{\star}}-1\right)^{2}}, \\
r & \simeq \frac{64}{N}\left(\frac{1+n\left(1-f_{\mathcal{R}_{\star}}\right)}{f_{\mathcal{R}_{\star}}-1}\right)^{2} .
\end{aligned}
$$

Since only $|n| \ll 1$ are allowed, as we see below, the results above, together with $a_{\mathrm{s}}$, can be further simplified as follows

$$
\begin{aligned}
n_{\mathrm{s}} & \simeq 1-\frac{2}{\widehat{N}_{\star}}-\frac{4 n}{N}-\frac{8 n^{2} \widehat{N}_{\star}}{3 N^{2}}, \\
r & \simeq \frac{4 N}{\widehat{N}_{\star}^{2}}-\frac{16 n^{3}}{N}+\frac{80 n^{2}}{3 N}-\frac{64 n^{3} \widehat{N}_{\star}}{3 N^{2}}, \\
a_{\mathrm{s}} & \simeq-\frac{2}{\widehat{N}_{\star}^{2}}+\frac{3 n}{\widehat{N}_{\star}^{2}}+\frac{8 n^{2}}{3 N^{2}}-\frac{7 N}{2 \widehat{N}_{\star}^{3}},
\end{aligned}
$$

where, for $n=0$, the well-known predictions of the Starobinsky model are recovered, i.e., $n_{\mathrm{s}} \simeq 0.966$ and $r=0.0032[r=0.0022]$ for $K=K_{1 \mathcal{R}}\left[K=K_{2 \mathcal{R}}\right]$. On the other hand, contributions proportional to $\widehat{N}_{\star}$ can be tamed for sufficiently low $n$ as we can verify numerically.

(b) For $K=K_{1}, K_{2}$ and $K_{3}$. Working along the lines of the previous paragraph we estimate the slow-roll parameters as follows

$$
\begin{aligned}
& \widehat{\epsilon}=\frac{8\left(1+n-n c_{+} \phi^{2}\right)^{2}}{c_{-} \phi^{2} f_{\mathrm{W}}^{2}} ; \\
& \frac{\widehat{\eta}}{4}=\frac{5+9 n-(3+10 n) c_{+} \phi^{2}+4 n^{2} f_{\mathrm{W}}^{2}+n c_{+}^{2} \phi^{2}}{c_{-} \phi^{2} f_{\mathrm{W}}^{2}} .
\end{aligned}
$$


Given that $\phi \ll 1$, Eq. (39a) is saturated at the maximal $\phi$ value, $\phi_{\mathrm{f}}$, from the following two values

$\phi_{1 \mathrm{f}} \simeq \sqrt{\frac{2}{c_{-}}} \frac{1}{r_{ \pm}^{1 / 3}}$ and $\phi_{2 \mathrm{f}} \simeq \sqrt{\frac{2}{c_{-}}}\left(\frac{3}{r_{ \pm}}\right)^{1 / 4}$,

where $\phi_{1 \mathrm{f}}$ and $\phi_{2 \mathrm{f}}$ are such that $\widehat{\epsilon}\left(\phi_{1 \mathrm{f}}\right) \simeq 1$ and $\widehat{\eta}\left(\phi_{2 \mathrm{f}}\right) \simeq$ 1 . The $n$ dependence is not so crucial for this estimation. Since $\phi_{\star} \gg \phi_{\mathrm{f}}$, from Eq. (40) we find

$\widehat{N}_{\star} \simeq \begin{cases}c_{-} \phi_{\star}^{2}\left(c_{-} r_{ \pm} \phi_{\star}^{2} / 2-1\right) / 8 & \text { for } n=0, \\ -\left(n c_{+} \phi_{\star}^{2}+\ln \left(1-\frac{n c_{+} \phi_{\star}^{2}}{1+n}\right)\right) / 8 n^{2} r_{ \pm} & \text {for } n \neq 0,\end{cases}$

where $\widehat{\phi}_{\star}$ is the value of $\widehat{\phi}$ when $k_{\star}$ crosses the inflationary horizon. As regards the consistency of the relation above for $n>0$, we note that we get $n c_{+} \phi_{\star}^{2}<1+n$ in all relevant cases and so, $\ln \left(1-n c_{+} \phi_{\star}^{2} /(1+n)\right)<0$ assures the positivity of $\widehat{N}_{\star}$. Solving the equations above w.r.t $\phi_{\star}$, we can express $\phi_{\star}$ in terms of $\widehat{N}_{\star}$ as follows

$\phi_{\star} \simeq \frac{f_{\mathcal{R}_{\star}}^{\frac{1}{2}}}{c_{+}^{\frac{1}{2}}}$ with $f_{\mathcal{R}_{\star}}= \begin{cases}1+\left(1+16 r_{ \pm} \widehat{N}_{\star}\right)^{\frac{1}{2}} & \text { for } n=0, \\ \left(1+n+W_{k}(y)\right) / n & \text { for } n \neq 0,\end{cases}$

where we make use of Eq. (23d). Also, $W_{k}$ is the Lambert $W$ (or product logarithmic) function [96] with

$y=-(1+n) \exp \left(-1-n\left(1+8 n \widehat{N}_{\star} r_{ \pm}\right)\right)$.

We take $k=0$ for $n>0$ and $k=-1$ for $n<0$.

Contrary to what happens for $K=K_{1 \mathcal{R}}$ and $K_{2 \mathcal{R}}, c_{-}$is not uniquely determined here. Therefore, for any $n$ we are obliged to impose a lower bound on it, above which $\phi_{\star} \leq 1$. Indeed, from Eq. (54) we have

$\phi_{\star} \leq 1 \Rightarrow c_{-} \geq f_{\mathcal{R}_{\star}} / r_{ \pm}$,

and so our proposal can be stabilized against corrections from higher order terms. Despite the fact that $c_{-}$may take relatively large values, the corresponding effective theories are valid [24-26] up to $m_{\mathrm{P}}=1$ for $r_{ \pm}$given by Eq. (38). To further clarify this point we have to identify the ultraviolet cut-off scale $\Lambda_{\mathrm{UV}}$ of the theory by analyzing the small-field behavior of our models. More specifically, adapting the expansions in Eqs. (37a) and (37b) in our present case, we end up with the expressions

$J^{2} \dot{\phi}^{2} \simeq\left(1-2 \bar{r}_{ \pm}^{3} \widehat{\delta \phi}+3 N \bar{r}_{ \pm}^{4} \widehat{\delta \phi}^{2}-4 N \bar{r}_{ \pm}^{5} \widehat{\delta \phi}^{3}+\cdots\right) \widehat{\widehat{\delta \phi}}^{2}$ where we set $\bar{r}_{ \pm}=\sqrt{r_{ \pm} /\left(1+N r_{ \pm}\right)}$, and

$$
\begin{aligned}
\widehat{V}_{\mathrm{HI}} \simeq & \frac{\lambda^{2} \bar{r}_{ \pm}^{2} \widehat{\delta \phi}^{2}}{4 c_{+}^{2}}\left(1-(3+4 n) \bar{r}_{ \pm} \widehat{\delta \phi}\right. \\
& \left.+\left(\frac{25}{4}+14 n+8 n^{2}\right) \bar{r}_{ \pm}^{2} \widehat{\delta \phi}^{2}+\cdots\right) .
\end{aligned}
$$

From the expressions above we conclude that $\Lambda_{\mathrm{UV}}=$ $m_{\mathrm{P}}$ since $r_{ \pm} \leq 1$ (and so $\bar{r}_{ \pm} \leq 1$ ) due to Eq. (38).

From the second equation in Eq. (40) we can also conclude that $\lambda$ is proportional to $c_{-}$for fixed $n$. Indeed, plugging Eq. (54) into this equation and solving w.r.t $\lambda$, we find

$\lambda=32 \sqrt{3 A_{\mathrm{s}}} \pi c_{-} r_{ \pm}^{3 / 2} f_{\mathcal{R} \star}^{n+1 / 2} \frac{n\left(1-f_{\mathcal{R} \star}\right)+1}{\left(f_{\mathcal{R} \star}-1\right)^{2}}$.

Numerically, - see below - we find that $\lambda / c_{-}$develops a maximum at $n \simeq-0.15$ which signals a transition to a branch of inflationary solutions which deviate from those obtained within the Starobinsky-like inflation. Inserting $f_{\mathcal{R} \star}$ from Eq. (54) into Eqs. (42a) and (42b) we obtain

$$
\begin{aligned}
& n_{\mathrm{s}} \simeq 1-\frac{8}{f_{\mathcal{R}_{\star}}}\left(\frac{3 f_{\mathcal{R}_{\star}}+1}{\left(f_{\mathcal{R}_{\star}}-1\right)^{2}}-n \frac{f_{\mathcal{R}_{\star}}+3}{f_{\mathcal{R}_{\star}}-1}+2 n^{2}\right) \text {, } \\
& r \simeq 128 \frac{r_{ \pm}}{f_{\mathcal{R}_{\star}}}\left(\frac{1-n\left(f_{\mathcal{R} \star}-1\right)}{f_{\mathcal{R}_{\star}}-1}\right)^{2}, \\
& a_{\mathrm{S}} \simeq \frac{64 r_{ \pm}^{2}}{3\left(f_{\mathcal{R} \star}-1\right)^{4} f_{\mathcal{R} \star}^{2}}\left(3-9 f_{\mathcal{R}_{\star}}\left(2 f_{\mathcal{R}_{\star}}+1\right)\right. \\
& +3\left(f_{\mathcal{R} \star}-1\right)\left(f_{\mathcal{R} \star}\left(7 f_{\mathcal{R} \star}+9\right)-4\right) n \\
& \left.+2\left(f_{\mathcal{R} \star}-1\right)^{2}\left(f_{\mathcal{R} \star}\left(f_{\mathcal{R}_{\star}}-42\right)+121\right) n^{2}\right) \text {. }
\end{aligned}
$$

where we can recognize the similarities with the formulas given in Eqs. (49a) and (49b). For $|n|<0.1$ these formulas may be expanded successively in series of $n$ and $1 / \widehat{N}_{\star}$ with results

$n_{\mathrm{s}} \simeq 1-\frac{16}{3} n^{2} r_{ \pm}-2 n \frac{r_{ \pm}^{1 / 2}}{\widehat{N}_{\star}^{1 / 2}}-\frac{3-2 n}{2 \widehat{N}_{\star}}-\frac{3+5 n}{24\left(\widehat{N}_{\star}^{3} r_{ \pm}\right)^{1 / 2}}$,

$r \simeq-\frac{8 n}{\widehat{N}_{\star}}-\frac{1}{2 \widehat{N}_{\star}^{2} r_{ \pm}}+\frac{2(3+2 n)}{3\left(\widehat{N}_{\star}^{3} r_{ \pm}\right)^{1 / 2}}+\frac{32 n^{2} r_{ \pm}^{1 / 2}}{3 \widehat{N}_{\star}^{1 / 2}}$

$a_{\mathrm{s}} \simeq-\frac{n r_{ \pm}^{1 / 2}}{\widehat{N}_{\star}^{3 / 2}}-\frac{3-2 n}{2 \widehat{N}_{\star}^{2}}$ 


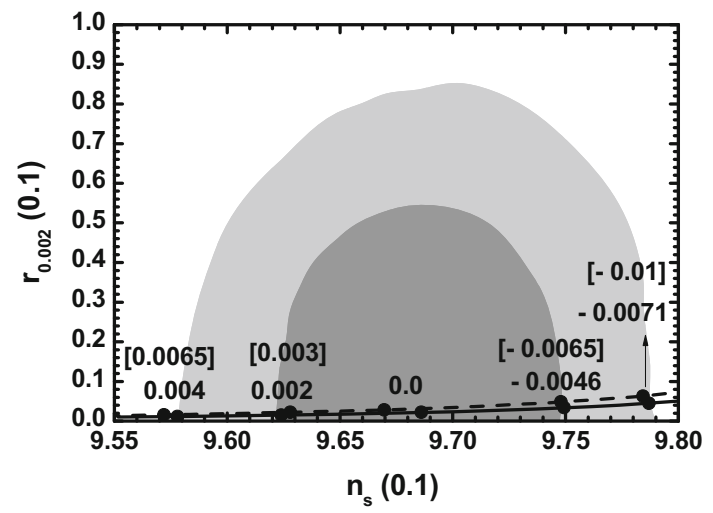

Fig. 1 Allowed curves in the $n_{\mathrm{s}}-r_{0.002}$ plane for $K=K_{1 \mathcal{R}}$ (dashed line) and $K=K_{2 \mathcal{R}}$ (solid line) - the $n$ values in [outside] squared brackets correspond to $K=K_{1 \mathcal{R}}\left[K=K_{2 \mathcal{R}}\right]$. The marginalized joint $68 \%$ [95\%] regions from Planck, BAO and BK14 data are depicted by the dark [light] shaded contours

From the expressions above, we can infer that there is a clear $n$ (and $r_{ \pm}$) dependence of the observables which deviate somewhat from those obtained in the pure Starobinsky-type inflation (or IG inflation) [4,5,7-12]. Note that the formulae, although similar, are not identical with those found in Ref. [35].

\subsubsection{Numerical results}

The approximate analytic expressions above can be verified by the numerical analysis. Namely, we apply the accurate expressions in Eq. (40) and confront them with the requirements in Eqs. (41a)-(41b) adjusting $c_{\mathcal{R}}$ and $\lambda$ for $K=K_{1 \mathcal{R}}$ and $K_{2 \mathcal{R}}$ or $c_{-}$and $\lambda$ for with any selected $n$. Then, we compute the model predictions via Eqs. (42a) and (42b). Our results are mainly displayed in Figs. 1 and 2, where we show a comparison of the models' predictions against the observational data $[23,59]$ in the $n_{\mathrm{s}}-r_{0.002}$ plane, where $r_{0.002}=16 \widehat{\epsilon}\left(\widehat{\phi}_{0.002}\right)$ with $\widehat{\phi}_{0.002}$ being the value of $\widehat{\phi}$ when the scale $k=0.002 / \mathrm{Mpc}$, which undergoes $\widehat{N}_{0.002}=\widehat{N}_{\star}+3.22$ e-foldings during IGHI, crosses the horizon of IGHI. Let us discuss separately the results for the two classes of models. In particular:

(a) For $K=K_{1 \mathcal{R}}$ and $K_{2 \mathcal{R}}$. We depict in Fig. 1 by a dashed [solid] line the model predictions for $K=K_{1 \mathcal{R}}$ [ $K=K_{2 \mathcal{R}}$ ] against the observational data. We see that the whole observationally favored range at low $r$ 's is covered varying $n$ which remains, though, rather close to zero. In fact $n$ is tuned closer to zero and $r$ is slightly lower compared to those obtained for $K=K_{1}, K_{2}$ and $K_{3}$ - see below. More explicitly, we find the allowed ranges

$$
0.9 \gtrsim n / 0.01 \gtrsim-1 \text { and } 1.5 \lesssim r / 10^{-3} \lesssim 6.6
$$

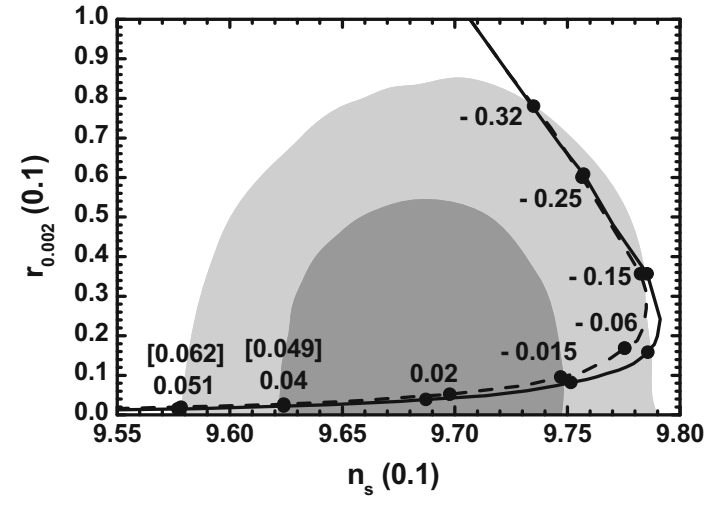

Fig. 2 The same as Fig. 1 but for $K=K_{1}$ (dashed line) and $K=K_{2}$ or $K_{3}$ (solid line) with the $n$ values indicated on the curves (the $n$ values in squared brackets correspond to $K=K_{1}$ )

for $K=K_{1 \mathcal{R}}$, whereas for $K=K_{2 \mathcal{R}}$ we have

$5.1 \gtrsim n / 0.001 \gtrsim-9$ and $1.1 \lesssim r / 10^{-3} \lesssim 5.9$

As $n$ varies in its allowed ranges presented below, we obtain

$2.3 \lesssim \lambda / 0.1 \lesssim 4$ or $1.9 \lesssim \lambda / 0.1 \lesssim 3.5$

for $K=K_{1 \mathcal{R}}$ or $K=K_{2 \mathcal{R}}$ respectively. If we take $n=0$, we find the central values of $\lambda$ in the ranges above which are 0.29 and 0.24 correspondingly.

(b) For $K=K_{1}, K_{2}$ and $K_{3}$. In this case, let us clarify that the (theoretically) free parameters of our models are $n$ and $\lambda / c_{-}$and not $n, c_{-}$, and $\lambda$ as naively expected recall that $M$ and $r_{ \pm}$are found from Eqs. (20) and (38). Indeed, if we perform the rescalings

$\Phi \rightarrow \Phi / \sqrt{c_{-}}, \quad \bar{\Phi} \rightarrow \bar{\Phi} / \sqrt{c_{-}}$and $S \rightarrow S$

$W$ in Eq. (1) depends on $\lambda / c_{-}$and $r_{ \pm}^{-1}$, while the $K$ 's in Eqs. (6a)-(6c) depend on $n$ and $r_{ \pm}$. As a consequence, $\widehat{V}_{\mathrm{HI}}$ depends exclusively on $\lambda / c_{-}$and $n$. Since the $\lambda / c_{-}$ variation is rather trivial - see Eq. (58) - we focus below on the variation of $n$.

In Fig. 2 we depict the theoretically allowed values with solid and dashed lines for $K=K_{2}$ or $K_{3}$ and $K=$ $K_{1}$ respectively. The variation of $n$ is shown along each line. In squared brackets we display the $n$ values for $K=K_{1}$ when these differ appreciably from those for $K=K_{2}$ or $K_{3}$. We remark that for $n>0$ there is a discrepancy of about $20 \%$ changing $K$ from $K_{1}$ to $K_{2}$ or $K_{3}$, which decreases as $n$ decreases below zero. This effect originates from the difference in $J$ - see Eqs. (31) and (33a) - which becomes smaller and smaller as $n$ decreases or $c_{-}$increases. We observe that $n>0$ values 
Table 2 Parameters and observables for the points shown in Fig. 2 with $K=K_{2}$ and $K_{3}$

\begin{tabular}{llllll}
\hline$n / 0.1$ & $r_{ \pm} / 0.1$ & $\lambda / 10^{-5} c_{-}$ & $n_{\mathrm{s}} / 0.1$ & $-a_{\mathrm{s}} / 10^{-4}$ & $r / 0.01$ \\
\hline 0.51 & 4.76 & 1.7 & 9.58 & 5.2 & 0.17 \\
0.4 & 4.81 & 2 & 9.62 & 5.2 & 0.25 \\
0.2 & 4.9 & 2.55 & 9.69 & 5 & 0.43 \\
-0.15 & 5.07 & 3.4 & 9.75 & 4.5 & 0.88 \\
-0.6 & 5.32 & 4.1 & 9.78 & 3.5 & 1.7 \\
-1.5 & 5.88 & 4.5 & 9.78 & 3.98 & 3.7 \\
-2.5 & 6.66 & 3.9 & 9.76 & 4 & 6.3 \\
-3.2 & 7.35 & 3.3 & 9.73 & 4.4 & 8.3 \\
\hline
\end{tabular}

dominate the part of the curves with lower $r$ values and $n_{\mathrm{s}} \leq 0.973$, whereas the $n<0$ values generate the part of the curves with $n_{\mathrm{S}}$ close to its upper bound in Eq. (43) and appreciably larger $r$ values. Roughly speaking, the displayed curves can be produced interconnecting the limiting points of the various curves in Fig. 2a of Ref. [35], although the curves for $0<n<0.1$ and $n<-0.1$ are not depicted there. This is because the $r_{ \pm}$'s resulting from Eq. (38) are close to their upper limits induced by Eq. (32).

Comparing these theoretical outputs with data depicted by the dark [light] shaded contours at $68 \%$ c.1. [95\% c.1.] we find the allowed ranges. Especially, for $K=K_{1}$ we obtain

$0.62 \gtrsim n / 0.1 \gtrsim-3.2, \quad 3.2 \lesssim r_{ \pm} / 0.1 \lesssim 4.16$

On the other hand, for $K=K_{2}$ or $K_{3}$, we find one branch localized in the ranges

$0.51 \gtrsim n / 0.1 \gtrsim-0.6, \quad 4.76 \lesssim r_{ \pm} / 0.1 \lesssim 5.32$,

and another one

$-1.5 \gtrsim n / 0.1 \gtrsim-3.2, \quad 5.88 \lesssim r_{ \pm} / 0.1 \lesssim 7.35$.

The findings for $K=K_{2}$ or $K_{3}$, can also be read-off from Table 2 where we list the values of the input parameter $(n)$ depicted in Fig. 2, the corresponding output parameters $\left(r_{ \pm}\right.$and $\left.\lambda / c_{-}\right)$and the inflationary observables. We observe that $n_{\mathrm{S}}$ and $r$ are well confined in the allowed regions of Eq. (43), while $a_{\mathrm{s}}$ varies in the range $-(3.98-5.2) \cdot 10^{-4}$ and so, our models are consistent with the fitting of data with the $\Lambda \mathrm{CDM}+r$ model [23]. Comparing these numerical values with those obtained by the analytic expressions in Eqs. (59a)-(59c) we obtain complete agreement for any $n$. On the other hand, the approximate formulas in Eqs. (60a)-(60c) are valid only

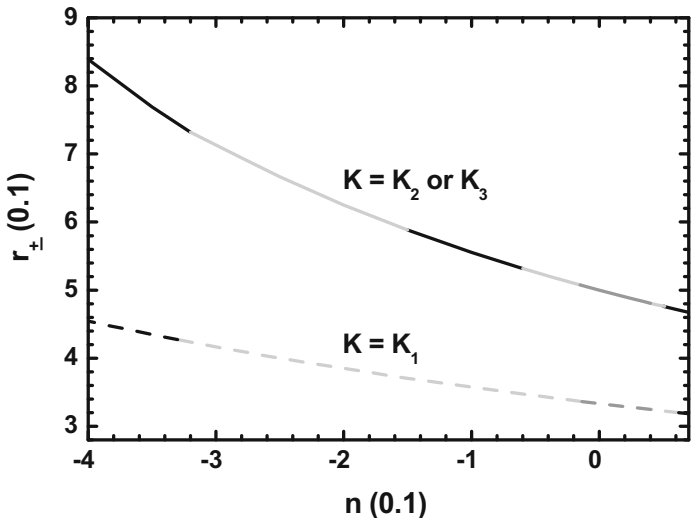

Fig. 3 Values of $r_{ \pm}$allowed by Eq. (20) as a function of $n$ for $K=K_{1}$ (dashed lines) and $K=K_{2}$ or $K_{3}$ (solid lines). The values which are also preferred by the observational data at $68 \%$ c.1. [ $95 \%$ c.1.] are included in the dark [light] gray segments

for $\left|n_{\mathrm{s}}\right|<0.1$, i.e., the Starobinsky-like region. Hilltop IGHI is attained for $n>0$ and there we find $\Delta_{\max \star} \gtrsim 0.155$, where $\Delta_{\max \star}$ increases as $n$ drops. The required tuning is not severe, mainly for $n<0.04$ since $\Delta_{\max \star} \gtrsim 20 \%$. Since our models predict $r \gtrsim 0.0017$, they are testable by the forthcoming experiments, like BICEP3 [60], PRISM [61], LiteBIRD [62] and CORE [63], which are expected to measure $r$ with an accuracy of $10^{-3}$. We do not present in Table $2 \phi_{\star}$ values since, as inferred by Eq. (58), every $\phi_{\star}$ satisfying Eq. (41a) leads to the same ratio $\lambda / c_{-}$. For the reasons mentioned below Eq. (56), we prefer $\phi_{\star} \leq 1$. To achieve this, we need $c_{-} \gtrsim(30-140)$ for $K=K_{1}$ and $c_{-} \gtrsim(40-160)$ for $K=K_{2}$ or $K_{3}$, where the variation of $c_{-}$is given as $n$ decreases.

For $K=K_{1}$ we expect similar values for $\lambda / c_{-}$and the inflationary observables. However, $r_{ \pm}$will differ appreciably due to the different relation between $n$ and $N-$ see Eq. (23f). To highlight it further, we present in Fig. 3 the $r_{ \pm}$values, obtained by Eq. (38), as a function of $n$ for $K=K_{1}$ (dashed lines) or $K=K_{2}$ and $K_{3}$ (solid lines). The values of the curves which are preferred by the observational data at $68 \%$ c.l. [95\% c.1.] are included in the dark [light] gray segments - cf. Fig. 2. We observe that for tiny $n$ values, $r_{ \pm}$which is roughly $1 / N$ lies close to $1 / 3$ for $K=K_{1}$ and $1 / 2$ for $K=K_{2}, K_{3}$. For larger $|n|$ values $r_{ \pm}$deviates more drastically from these values. The rather different predictions attained for low $(|n| \leq$ $0.1)$ and large $(|n|>0.1) n$ values hint that the structure of $\widehat{V}_{\mathrm{HI}}$ changes drastically. To illuminate this fact we show $\widehat{V}_{\mathrm{HI}}$ as a function of $\phi$ in Fig. 4 for $K=K_{2}$ or $K_{3}, n=0.02$ (gray line) and $n=-0.25$ (light gray line). We take in both cases $\phi_{\star}=1$. Therefore, the corresponding $c_{-}$and $\lambda$ values are confined to their lowest possible values enforcing Eqs. (41a) and (41b). More 


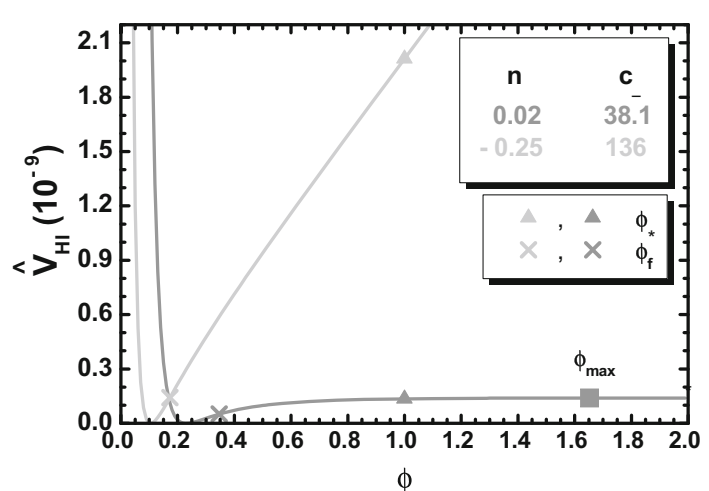

Fig. 4 The inflationary potential $\widehat{V}_{\mathrm{HI}}$ as a function of $\phi$ for $K=K_{2}$ or $K_{3}, \phi_{\star}=1$ and $n=0.02$ (gray line) or $n=-0.25$ (light gray line). The values corresponding to $\phi_{\star}, \phi_{\mathrm{f}}$ and $\phi_{\max }$ (for $n=0.02$ ) are also indicated

specifically, we find $\lambda=9.7 \cdot 10^{-4}$ or $5.3 \cdot 10^{-3}$ and $c_{-}=38.1$ or 136 , with $M=0.23$ or $M=0.105$ for $n=0.02$ or $n=-0.25$ respectively. The corresponding $r_{ \pm}$values and observable quantities are listed in Table 2 . We see that in both cases $\widehat{V}_{\mathrm{HI}}$ develops a singularity at $\phi=0$ contrary to the models of non-minimal inflation - cf. Refs. [32,35] - where $\widehat{V}_{\mathrm{HI}}$ exhibits a maximum. However, for $n>0$ and $|n| \sim 0.01, \widehat{V}_{\mathrm{HI}}$ resembles the potential of Starobinsky model with a maximum at $\phi_{\max }=1.65$. This does not affect much the inflationary evolution since we find $\Delta_{\max \star}=39 \%$, and so the tuning of the initial conditions of IGHI is rather mild. On the contrary, $\widehat{V}_{\mathrm{HI}}$ increases monotonically and almost linearly with $\phi$ for $n=-0.25$. Both behaviors can be interpreted from Eq. (24) taking into account that $f_{\mathrm{W}} \sim \phi^{2}$ and $f_{\mathcal{R}} \sim \phi^{2}$. For $n \sim 0.01, \widehat{V}_{\mathrm{HI}} \sim f_{\mathrm{W}}^{2} / f_{\mathcal{R}}^{2}$ becomes more or less constant, whereas for $n \simeq-0.25$, $\widehat{V}_{\mathrm{HI}} \sim f_{\mathrm{W}}^{2} / f_{\mathcal{R}}^{2 \cdot 3 / 4} \sim \phi^{4} / \phi^{3} \sim \phi$. It is also remarkable that in the latter case $r$ increases, thanks to the increase of the inflationary scale, $\widehat{V}_{\mathrm{HI}}^{1 / 4}$. Similar region of parameters is recently reported in Ref. [98].

\section{A possible post-inflationary completion}

Our discussion about IGHI is certainly incomplete without at least mentioning how the transition to the radiation dominated era is realized and the observed BAU is generated. Since these goals are related to the possible decay channels of the inflaton, the connection of IGHI with some low energy theory is unavoidable. A natural, popular and well motivated framework for particle physics at the $\mathrm{TeV}$ scale beyond the standard model is MSSM. A possible route to such a more complete scenario is described in Sect. 3.1. Next, Sect. 3.2 is devoted to the connection of IGHI with MSSM through the generation of the $\mu$ term. In Sect. 3.3, finally, we analyze the scenario of nTL exhibiting the relevant constraints and further restricting the parameters. Here and hereafter we restore units, i.e., we take $m_{\mathrm{P}}=2.433 \cdot 10^{18} \mathrm{GeV}$.

\subsection{The relevant set-up}

Following the post-inflationary setting of Ref. [35] we supplement the superpotential of the theory with the terms

$W_{\mathrm{RHN}}=\lambda_{i N^{c}} \bar{\Phi} N_{i}^{c 2}+h_{N i j} N_{i}^{c} L_{j} H_{u}$,

which allows for the implementation of type I see-saw mechanism (providing masses to light neutrinos) and supports a robust baryogenesis scenario through nTL, and

$W_{\mu}=\lambda_{\mu} S H_{u} H_{d}$,

inspired by Ref. [37], which offers a solution to one of the most tantalizing problems of MSSM, namely the generation of a $\mu$ term - for an alternative solution see Ref. [99]. Here we adopt the notation and the $B-L$ and $\mathrm{R}$ charges of the various superfields as displayed in Table 1 of Ref. [35]. Let us only note that $L_{i}$ denotes the $i$-th generation $S U(2)_{\mathrm{L}}$ doublet left-handed lepton superfields, and $H_{u}\left[H_{d}\right]$ is the $S U(2)_{\mathrm{L}}$ doublet Higgs superfield which couples to the up [down] quark superfields. Also, we assume that the superfields $N_{j}^{c}$ have been rotated in the family space so that the coupling constants $\lambda_{i}$ are real and positive. This is the so-called [4,5, 35] $N_{i}^{c}$ basis, where the $N_{i}^{c}$ masses, $M_{i N^{c}}$, are diagonal, real, and positive.

We assume that the extra fields $X^{\beta}=H_{u}, H_{d}, \widetilde{N}_{i}^{c}$ have identical kinetic terms as the stabilizer field $S$ expressed by the functions $F_{l S}$ with $l=1,2,3$ in Eqs. (9a)-(9c)-see Ref. [35]. Therefore, $N_{S}$ may be renamed $N_{X}$ henceforth. The inflationary trajectory in Eq. (22) has to be supplemented by the conditions

$H_{u}=H_{d}=\widetilde{N}_{i}^{c}=0$

and the stability of this path has to be checked, parameterizing the complex fields above as we do for $S$ in Eq. (21). The relevant masses squared are listed in Table 3 for $K=K_{1 \mathcal{R}}$ and $K_{2 \mathcal{R}}$ and Table 4 for $K=K_{1}, K_{2}$ and $K_{3}$, where we see that $\widehat{m}_{i v}^{2}>0$ for every $\phi$. On the other hand, the positivity of the mass-squared eigenvalues $\widehat{m}_{h-}^{2}$ associated with the eigenstates $\widehat{h}_{-}$and $\widehat{\bar{h}}_{-}$, where

$\widehat{h}_{ \pm}=\left(\widehat{h}_{u} \pm \widehat{h}_{d}\right) / \sqrt{2}$ and $\widehat{\bar{h}}_{ \pm}=\left(\widehat{\bar{h}}_{u} \pm \widehat{\bar{h}}_{d}\right) / \sqrt{2}$

with the hatted fields being defined as $\hat{s}$ and $\hat{\bar{s}}$ in Eq. (33b), requires the establishment of the inequalities 
Table 3 Mass-squared spectrum of the non-inflaton sector for $K=K_{1 \mathcal{R}}$ and $K_{2 \mathcal{R}}$ along the path in Eqs. (22) and (68)

\begin{tabular}{|c|c|c|c|c|}
\hline \multirow[t]{2}{*}{ Fields } & \multirow[t]{2}{*}{ Eigenstates } & \multicolumn{3}{|c|}{ Masses squared } \\
\hline & & & $K=K_{1 \mathcal{R}}$ & $K=K_{2 \mathcal{R}}$ \\
\hline 10 Real & $\widehat{h}_{ \pm}, \widehat{\bar{h}}_{ \pm}$ & $\widehat{m}_{h \pm}^{2}$ & $3 \widehat{H}_{\mathrm{HI}}^{2}\left(\phi^{2} / 2 N \pm 2 \lambda_{\mu} / \lambda\right)$ & $3 \widehat{H}_{\mathrm{HI}}^{2}\left(1+1 / N_{X} \pm 4 \lambda_{\mu} / \lambda \phi^{2}\right)$ \\
\hline Scalars & $\widehat{\widetilde{v}}_{i}^{c}, \widehat{\tilde{\tilde{v}}}_{i}^{c}$ & $\widehat{m}_{i \tilde{v}^{c}}^{2}$ & $3 \widehat{H}_{\mathrm{HI}}^{2}\left(\phi^{2} / 2 N+8 \lambda_{i N^{c}}^{2} / \lambda^{2}\right)$ & $3 \widehat{H}_{\mathrm{HI}}^{2}\left(1+1 / N_{X}+16 \lambda_{i N^{c}}^{2} / \lambda^{2} \phi^{2}\right)$ \\
\hline 3 Weyl Spinors & $\widehat{N}_{i}^{c}$ & $\widehat{m}_{i N^{c}}^{2}$ & \multicolumn{2}{|c|}{$48 \widehat{H}_{\mathrm{HI}}^{2} \lambda_{i N c}^{2} / \lambda^{2} \phi^{2}$} \\
\hline
\end{tabular}

Table 4 The same as Table 3 but for $K=K_{1}, K_{3}$ and $K_{3}$

\begin{tabular}{lllll}
\hline Fields & Eigenstates & \multicolumn{3}{c}{ Masses squared } \\
\cline { 3 - 5 } & & & $K=K_{1}$ & $K=K_{2}$ \\
\hline 10 Real & $\widehat{h}_{ \pm}, \widehat{\bar{h}}_{ \pm}$ & $\widehat{m}_{h \pm}^{2}$ & $3 \widehat{H}_{\mathrm{HI}}^{2}\left(1+f_{\mathrm{W}} / N \pm 4 \lambda_{\mu} c_{+}^{2} \phi^{2} / \lambda f_{\mathrm{W}}\right)$ & $3 \widehat{H}_{\mathrm{HI}}^{2}\left(1+1 / N_{X} \pm 4 \lambda_{\mu} c+\lambda f_{\mathrm{W}}\right)$ \\
Scalars & $\widehat{\tilde{v}}_{i}^{c}, \widehat{\tilde{\tilde{v}}}_{i}^{c}$ & $\widehat{m}_{i \tilde{v}^{c}}^{2}$ & $3 \widehat{H}_{\mathrm{HI}}^{2}\left(\left(1+f_{\mathrm{W}} / N\right) / c_{+} \phi^{2}+16 \lambda_{i N^{c}}^{2} c_{+}^{2} \phi^{2} / \lambda^{2} f_{\mathrm{W}}^{2}\right)$ & $3 \widehat{H}_{\mathrm{HI}}^{2}\left(1+1 / N_{X}+16 \lambda_{i N^{c}}^{2} c_{+}^{2} \phi^{2} / \lambda^{2} f_{\mathrm{W}}^{2}\right)$ \\
3 Weyl Spinors & $\widehat{N}_{i}^{c}$ & $m_{i N^{c}}^{2}$ & $48 \lambda_{i N^{c}}^{2} c_{+}^{2} \phi^{2} \widehat{H}_{\mathrm{HI}}^{2} / \lambda^{2} f_{\mathrm{W}}^{2}$ \\
\hline
\end{tabular}

$$
\begin{array}{ll}
\lambda_{\mu} \lesssim \lambda \phi^{2} / 4 N & \text { for } K=K_{1 \mathcal{R}} \\
\lambda_{\mu} \lesssim \lambda \phi^{2}\left(1+1 / N_{X}\right) / 4 & \text { for } K=K_{2 \mathcal{R}} \\
\lambda_{\mu} \lesssim \lambda f_{\mathrm{W}}\left(1+f_{\mathrm{W}} / N\right) / 4 \lambda_{\mu} c_{+}^{2} \phi^{2} & \text { for } K=K_{1} \\
\lambda_{\mu} \lesssim \lambda f_{\mathrm{W}}\left(1+1 / N_{X}\right) / 4 c_{+} & \text {for } K=K_{2}, K_{3} .
\end{array}
$$

In all cases, the inequalities are fulfilled for $\lambda_{\mu} \lesssim 2 \cdot 10^{-5}$. Similar numbers are obtained in Refs. [4,5,35]. We do not consider such a condition on $\lambda_{\mu}$ as unnatural, given that the Yukawa coupling constant $h_{1 U}$, which provides masses to the up-type quarks, is of the same order of magnitude too at a high scale - cf. Ref. [100]. Note that the hierarchy in Eqs. (70a)-(70d) between $\lambda_{\mu}$ and $\lambda$ differs from that imposed in the models [37] of F-term hybrid inflation, where $S$ plays the role of inflaton and $\Phi, \bar{\Phi}, H_{u}$ and $H_{d}$ are confined at zero. Indeed, in that case we demand [37] $\lambda_{\mu}>\lambda$ so that the tachyonic instability in the $\Phi-\bar{\Phi}$ direction occurs first, and the $\Phi-\bar{\Phi}$ system start evolving towards its v.e.v, whereas $H_{u}$ and $H_{d}$ continue to be confined to zero. In our case, though, the inflaton is included in the $\bar{\Phi}-\Phi$ system while $S$ and the $H_{u}-H_{d}$ system are safely stabilized at the origin both during and after IGHI. Therefore, $\phi$ settles in its vacuum and $S, H_{u}$ and $H_{d}$ take their non-vanishing electroweak scale v.e.vs afterwards.

\subsection{A solution to the $\mu$ problem of MSSM}

A byproduct of the $R$ symmetry associated with our models is that it assists us to understand the origin of the $\mu$ term of
MSSM - see Sect. 3.2.1 - connecting thereby the high with the low energy phenomenology as described in Sect. 3.2.2.

\subsubsection{Generating the $\mu$ parameter}

Working along the lines of Sect. 2.1.2 we can verify that the presence of the terms in Eqs. (67a) and (67b) leave the v.e.vs in Eq. (19) unaltered whereas those of $X^{\beta}$ are found to be

$\left\langle H_{u}\right\rangle=\left\langle H_{d}\right\rangle=\left\langle\tilde{N}_{i}^{c}\right\rangle=0$.

On the other hand, the contributions from the soft SUSY breaking terms, although negligible during IGHI - since these are expected to be much smaller than $\phi-$, may slightly shift $[4,5,35,37]\langle S\rangle$ from zero in Eq. (19). Indeed, the relevant potential terms are

$$
V_{\text {soft }}=\left(\lambda A_{\lambda} S \bar{\Phi} \Phi-\mathrm{a}_{S} S \lambda M^{2} / 4+\text { h.c. }\right)+m_{\gamma}^{2}\left|X^{\gamma}\right|^{2},
$$

where $X^{\gamma}=\Phi, \bar{\Phi}, S, H_{u}, H_{d}, \widetilde{N}_{i}^{c}$, and $m_{\gamma}, A_{\lambda}$ and a $a_{S}$ are soft SUSY breaking mass parameters of the order of TeV. The emergence of these terms depend on the mechanism of SUSY breaking which is not specified here. We restrict ourselves to the assumption that this extra sector of the theory may be included in the present set-up without disturbing the status of inflation - cf. Refs. [101-103]. Confining $\Phi, \bar{\Phi}, H_{u}, H_{d}$ and $N_{i}^{c}$ in their v.e.vs in Eqs. (19) and (71), $\widehat{V}$ in Eq. (10b) reduces again to $V_{\text {eff }}$ in Eq. (15a) with vanishing terms represented by ellipsis since $\left\langle W_{\mathrm{HI}}\right\rangle=0$. We then rotate $S$ to the real axis by an appropriate $R$-transformation and choose conveniently the phases of $A_{\lambda}$ and as so as the total low-energy potential

$V_{\mathrm{tot}}=V_{\mathrm{eff}}+V_{\mathrm{soft}}$ 
to be minimized. Since the form of $V_{\text {eff }}$ depends on the adopted $K$, we single out the cases:

(a) $K=K_{1}, K_{2}$ and $K_{3}$. Focusing on $K=K_{2}$ or $K_{3}$ we obtain

$$
\left\langle V_{\mathrm{tot}}(S)\right\rangle \simeq \frac{\lambda^{2} S^{2}}{2\left\langle\operatorname{det} M_{ \pm}\right\rangle}\left(c_{-} M^{2}-N m_{\mathrm{P}}^{2}\right)-\lambda \mathrm{a}_{3 / 2} m_{3 / 2} M^{2} S
$$

where the first term in the r.h.s originates from the second line of Eq. (18) for $e^{\widetilde{K}_{+} / m_{\mathrm{P}}^{2}} \simeq 1$, and $\Phi$ and $\bar{\Phi}$ equal to their v.e.vs in Eq. (19). Also, we take into account that $m_{S} \ll M$, and we set

$\left|A_{\lambda}\right|+\left|\mathrm{a}_{S}\right|=2 \mathrm{a}_{3 / 2} m_{3 / 2}$,

where $m_{3 / 2}$ is the $\widetilde{G}$ mass and $\mathrm{a}_{3 / 2}>0$ a parameter of order unity which parameterizes our ignorance of the dependence of $\left|A_{\lambda}\right|$ and $\left|\mathrm{a}_{S}\right|$ on $m_{3 / 2}$. The minimization condition for the total potential in Eq. (74a) w.r.t $S$ leads to a non vanishing $\langle S\rangle$ as follows

$\frac{d}{d S}\left\langle V_{\text {tot }}(S)\right\rangle=0 \Rightarrow\langle S\rangle \simeq \mathrm{a}_{3 / 2} m_{3 / 2} c_{-}\left(1+N r_{ \pm}\right) / \lambda$

since from Eqs. (17c) (19) and (20) we infer

$\left\langle\operatorname{det} M_{ \pm}\right\rangle=c_{-}^{2}-N^{2} c_{+}^{2}=c_{-}^{2}\left(1+N r_{ \pm}\right)\left(1-N r_{ \pm}\right)$.

At this $S$ value, $\left\langle V_{\text {tot }}(S)\right\rangle$ develops a minimum since

$\frac{d^{2}}{d S^{2}}\left\langle V_{\text {tot }}(S)\right\rangle=\lambda^{2} / c_{+}\left(c_{-}+N c_{+}\right)>0$.

For $K=K_{1}$ Eq. (74c) can be obtained again by doing an expansion of the relevant expressions in powers $1 / m_{\mathrm{P}}$. The $\mu$ term generated from Eq. (67b) exhibits the mixing parameter

$\mu=\lambda_{\mu}\langle S\rangle \simeq \lambda_{\mu} \mathrm{a}_{3 / 2} m_{3 / 2} c_{-}\left(1+N r_{ \pm}\right) / \lambda$.

Comparing this result with the corresponding one in Ref. [35], we deduce a crucial difference regarding the sign of the expression in the parenthesis which originates from the terms in the second line of Eq. (18). With the aid of Eq. (58) we may eliminate $c_{-}$and $\lambda$ from the above result which then reads

$\mu \simeq 1.2 \cdot 10^{2} \lambda_{\mu} \frac{\mathrm{a}_{3 / 2} m_{3 / 2}\left(1+N r_{ \pm}\right)\left(f_{\mathcal{R}_{\star}}-1\right)^{2}}{r_{ \pm}^{3 / 2} f_{\mathcal{R}_{\star}}^{n+1 / 2}\left(n\left(1-f_{\mathcal{R}_{\star}}\right)+1\right)}$, where Eq. (41b) is employed to obtain the numerical prefactor. Taking into account Eqs. (38) and (54), we infer that the resulting $\mu$ depends only on $n$ and not on $\lambda, c_{-}$and $r_{ \pm}-$cf. Refs. [35,37]. For the $\lambda_{\mu}$ values allowed by Eqs. (70c) and (70d), any $|\mu|$ value is accessible with a mild hierarchy between $m_{3 / 2}$ and $\mu$ - from Table 4 we see that both signs of $\lambda_{\mu}$ (and so $\mu$ ) are possible without altering the stability analysis of the inflationary system. To understand this, let us first remark that Eq. (20) implies $r_{ \pm} \simeq 1 / N$ and $f_{\mathcal{R}_{\star}}$ varies from about 12 to 68 [15 to 119] for $K=K_{1}$ [ $K=K_{2}$ and $K_{3}$ ], as $n$ varies in the allowed ranges of Eqs. (64)-(66). A rough estimation gives $\mu \sim 10^{2} \lambda_{\mu} f_{\mathcal{R}_{\star}}^{3 / 2}=10^{-1.5} \mathrm{~m}_{3 / 2}$ and so we expect that $\mu$ is about one order of magnitude less than $m_{3 / 2}$.

(b) $K=K_{1 \mathcal{R}}$ and $K_{2 \mathcal{R}}$. In this case, $V_{\text {tot }}$ in Eq. (73) with all the fields except $S$ equal to their v.e.vs in Eqs. (19) and (71) is written as

$\left\langle V_{\mathrm{tot}}(S)\right\rangle=\frac{\lambda^{2} m_{\mathrm{P}}^{2} S^{2}}{c_{\mathcal{R}}\left(N c_{\mathcal{R}}-1\right)}-\lambda \mathrm{a}_{3 / 2} m_{3 / 2} M^{2} S$.

The minimization of $\left\langle V_{\text {tot }}(S)\right\rangle$ w.r.t $S$ leads to a new non vanishing $\langle S\rangle$,

$\langle S\rangle \simeq N \mathrm{a}_{3 / 2} m_{3 / 2} c_{\mathcal{R}} / \lambda$,

where $M$ is replaced by Eq. (20). Therefore, the $\mu$ parameter involved in Eq. (67b) is

$\mu=\lambda_{\mu}\langle S\rangle=N \lambda_{\mu} \mathrm{a}_{3 / 2} m_{3 / 2} c_{\mathcal{R}} / \lambda$.

This still depends only $n$ thanks to the condition in Eq. (48) which fixes $\lambda / c_{\mathcal{R}}$ as a function of $n-$ see Eq. (47).

To highlight further the conclusions above, we can employ Eq. (75a) to derive the $m_{3 / 2}$ values required so as to obtain a specific $\mu$ value. Given that Eq. (75a) depends on $n$, which crucially influences $n_{\mathrm{S}}$ and $r$, we expect that the required $m_{3 / 2}$ is a function of $n_{\mathrm{S}}$ and $r$ as depicted in Fig. 5a, b respectively. We take $\lambda_{\mu}=10^{-6}$, in accordance with Eqs. (70c) and (70d), $\mathrm{a}_{3 / 2}=1, K=K_{2}$ or $K_{3}$ with $N_{X}=2$ and $\mu=0.5 \mathrm{TeV}$ (dot-dashed line), $\mu=1 \mathrm{TeV}$ (solid line), or $\mu=2 \mathrm{TeV}$ (dashed line). Varying $n$ in the allowed range indicated in Fig. 2a we obtain the variation of $m_{3 / 2}$ solving Eq. (75a) w.r.t $m_{3 / 2}$. The values of the curves which are preferred by the observational data at $68 \%$ c.l. [95\% c.1.] are included in the dark [light] gray segments - cf. Fig. 2. We see that $m_{3 / 2}$ increases with $\mu$ and its lowest value $m_{3 / 2} \simeq 4 \mathrm{TeV}$ is obtained for $\mu=0.5 \mathrm{TeV}$. As we anticipated above, $m_{3 / 2}$ is almost one order of magnitude larger than the corresponding $\mu$. Moreover, for fixed $\mu$, each curve develops a maximum 


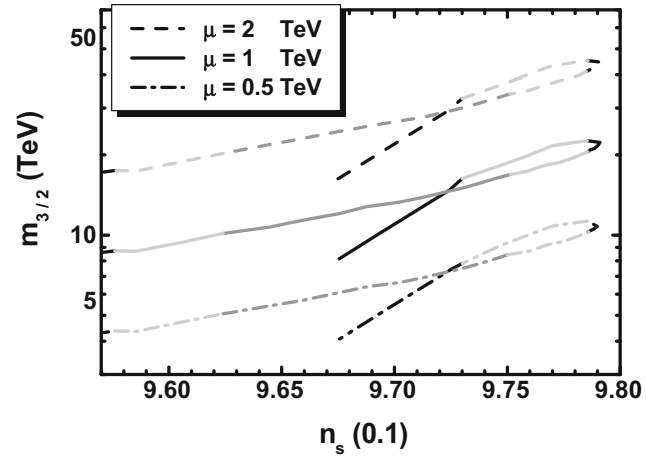

(a)

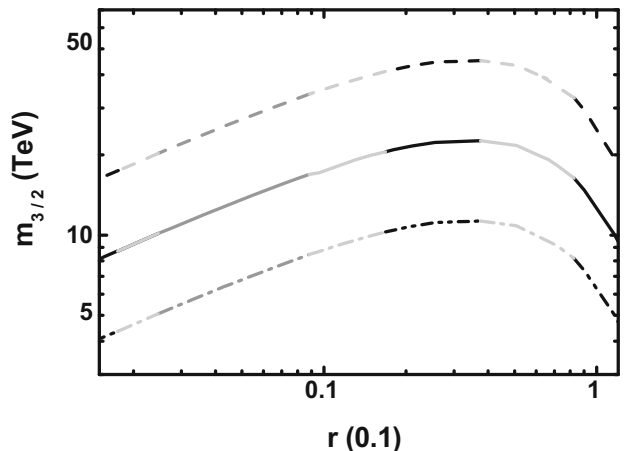

(b)

Fig. 5 The gravitino mass $m_{3 / 2}$ versus $n_{\mathrm{S}}$ (a) and $r$ (b) for $\lambda_{\mu}=10^{-6}$, a $3 / 2=1, K=K_{2}$ or $K_{3}$ with $N_{X}=2$ and $\mu=0.5$ TeV (dot-dashed line), $\mu=1 \mathrm{TeV}$ (solid line), or $\mu=2 \mathrm{TeV}$ (dashed line). The color coding is as in Fig. 3

at $n \simeq-0.15$, which coincides with the right corner of the curve in Fig. 2. This behavior deviates a lot from the one found in Ref. [35] and comes from the different sign in the parenthesis of Eq. (75a).

\subsubsection{Connection with the parameters of CMSSM}

The SUSY breaking effects, considered in Eq. (72), explicitly break $U(1)_{R}$ to a subgroup, $\mathbb{Z}_{2}^{R}$ which can be identified with a matter parity. Under this discrete symmetry all the matter (quark and lepton) superfields change sign - see Table 1 of Ref. [35]. Since $S$ has the $R$ symmetry of the total superpotential of the theory, $\langle S\rangle$ in Eq. (74c) also breaks spontaneously $U(1)_{R}$ to $\mathbb{Z}_{2}^{R}$. Thanks to this fact, $\mathbb{Z}_{2}^{R}$ remains unbroken and so no disastrous domain walls are formed. Combining $\mathbb{Z}_{2}^{R}$ with the $\mathbb{Z}_{2}^{\mathrm{f}}$ fermion parity, under which all fermions change sign, yields the well-known $R$-parity. This residual symmetry prevents rapid proton decay and guarantees the stability of the lightest SUSY particle (LSP), providing thereby a wellmotivated cold dark matter (CDM) candidate.

The candidacy of LSP may be successful, if it generates the correct CDM abundance [97] within a concrete low energy framework, which in our case is the MSSM or one of its variants - for an alternative approach within high-scale SUSY see Ref. [105]. Here, we adopt the Constrained MSSM (CMSSM) which is the most restrictive, predictive and wellmotivated version of MSSM, which allows the lightest neutralino to play the role of LSP in a sizable portion of the parametric space. This is based on the free parameters

$\operatorname{sign} \mu, \quad \tan \beta=\left\langle H_{u}\right\rangle /\left\langle H_{d}\right\rangle, M_{1 / 2}, m_{0}$, and $A_{0}$,

where $\operatorname{sign} \mu$ is the sign of $\mu$, and the three last mass parameters denote the common gaugino mass, scalar mass and trilinear coupling constant, respectively, defined (normally) at $M_{\mathrm{GUT}}$. The parameter $|\mu|$ is not free, since it is computed at low scale by enforcing the conditions for the electroweak symmetry breaking. The values of these parame-
Table 5 The required $\lambda_{\mu}$ values rendering our models compatible with the best-fit points of the CMSSM as found in Ref. [104] with the assumptions in Eq. (77).

\begin{tabular}{lclll}
\hline CMSSM region & $A / H$ funnel & \multicolumn{1}{l}{$\tilde{\tau}_{1}-\chi$} & $\tilde{t}_{1}-\chi$ & $\tilde{\chi}_{1}^{ \pm}-\chi$ \\
\cline { 3 - 5 } & \multicolumn{4}{l}{ Coannihilation } \\
\hline$A_{0} \mid(\mathrm{TeV})$ & 9.924 & 1.227 & 9.965 & 9.2061 \\
$m_{0}(\mathrm{TeV})$ & 9.136 & 1.476 & 4.269 & 9.000 \\
$|\mu|(\mathrm{TeV})$ & 1.409 & 2.62 & 4.073 & 0.983 \\
$\mathrm{a}_{3} / 2$ & 1.086 & 0.831 & 2.33 & 1.023 \\
$\lambda_{\mu}\left(10^{-6}\right)$ for $K=K_{1 \mathcal{R}}$ & & & \\
$\quad n=0$ & 0.963 & 14.48 & 2.91 & 0.723 \\
$\lambda_{\mu}\left(10^{-6}\right)$ for $K=K_{2 \mathcal{R}}$ & & & \\
$n=0$ & 1.184 & 17.81 & 3.41 & 0.89 \\
$\lambda_{\mu}\left(10^{-6}\right)$ for $K=K_{1}$ & & & \\
$n=0.02$ & 1.409 & 21.19 & 4.063 & 1.059 \\
$n=-0.25$ & 1.87 & 28.11 & 5.39 & 1.405 \\
$\lambda_{\mu}\left(10^{-6}\right)$ for $K=K_{2}$ and $K_{3}$ & & & \\
$n=0.02$ & 1.814 & 27.28 & 5.23 & 1.363 \\
$n=-0.25$ & 2.784 & 41.86 & 8.025 & 2.092 \\
\hline
\end{tabular}

ters can be tightly restricted imposing a number of cosmophenomenological constraints from which the consistency of LSP relic density with observations plays a central role. Some updated results are recently presented in Ref. [104], where we can also find the best-fit values of $\left|A_{0}\right|, m_{0}$ and $|\mu|$ listed in the first four lines of Table 5. We see that there are four allowed regions characterized by the mechanism applied for accommodating an acceptable CDM abundance.

Taking advantage of this investigation, we can check whether the $\mu$ and $m_{3 / 2}$ values satisfying Eq. (75a) are consistent with these values. Selecting some representative $n$ values and adopting the identifications

$m_{0}=m_{3 / 2}$ and $\left|A_{\lambda}\right|=\left|\mathrm{a}_{S}\right|=\left|A_{0}\right|$, 
we can first derive $a_{3 / 2}$ from Eq. (74b) and then the $\lambda_{\mu}$ values from Eqs. (75a)-(76c), which yield the phenomenologically desired $|\mu|$ shown in the third line of Table 5 . Here we assume that renormalization effects in the derivation of $\mu$ are negligible. The outputs of our computation are assembled in the last ten lines of Table 5. As inputs, we take $n=0.0$ for $K=K_{1 \mathcal{R}}$ and $K_{2 \mathcal{R}} n=0.02$ and -0.25 for $K=K_{1}, K_{2}$ and $K_{3}$. These are central values in the regions compatible with the inflationary observations as found in Sect. 2.3.3. The $\lambda_{\mu}$ values for $K=K_{1 \mathcal{R}}$ and $K_{2 \mathcal{R}}$ are lower than those obtained for $K=K_{1}, K_{2}$ and $K_{3}$, larger than those found in Ref. [35], and similar to those in Refs. [4,5] especially for $K=K_{1 \mathcal{R}}$. On the other hand, the $\lambda_{\mu}$ values found for $K=K_{1}, K_{2}$ and $K_{3}$ are larger compared to those found in Refs. [4,5,35].

From the outputs we infer that the required $\lambda_{\mu}$ values are comfortably compatible with Eqs. (70a)-(70d) for $N_{X}=2$, in all cases besides the one corresponding to the $\tilde{\tau}_{1}-\chi$ coannihilation region. In that case, $m_{0}$ is lower than $|\mu|$ and so marginally large $\lambda_{\mu}$ values are required. In the cases where numbers are written in italics, we obtain instability along the inflationary path for $K=K_{1 \mathcal{R}}$ and $K_{1}$, whereas for $K=$ $K_{2 \mathcal{R}}, K_{2}$ and $K_{3}$ we need $0 \leq N_{X} \leq 1$ to avoid this effect. In sharp contrast to the model of Ref. [35], only the $A / H$ funnel and $\tilde{\chi}_{1}^{ \pm}-\chi$ coannihilation regions can be consistent with the $\widetilde{G}$ limit on $T_{\mathrm{rh}}-$ see Sect. 3.3.2. Indeed, $m_{3 / 2} \gtrsim$ $9 \mathrm{TeV}$ become cosmologically safe under the assumption of an unstable $\widetilde{G}$, for the $T_{\text {rh }}$ values necessitated for satisfactory leptogenesis - see Sect. 3.3.3.

\subsection{Non-thermal leptogenesis}

Our next task is to specify how our inflationary scenario makes a transition to the radiation dominated era - see Sect. 3.3.1 - and offers an explanation of the observed BAU consistent with the $\widetilde{G}$ constraint and the low energy neutrino data - see Sect. 3.3.2. Our results are summarized in Sect. 3.3.3.

\subsubsection{Inflaton mass and decay}

When IGHI is over, the inflaton continues to roll down towards the SUSY vacuum, Eq. (19). Soon after, it settles into a phase of damped oscillations around the minimum of $\widehat{V}_{\mathrm{HI}}$. The (canonically normalized) inflaton,

$\widehat{\delta \phi}=\langle J\rangle \delta \phi$ with $\delta \phi=\phi-M$,

and

$$
\langle J\rangle= \begin{cases}\sqrt{N c_{\mathcal{R}}} & \text { for } K=K_{1 \mathcal{R}} \text { and } K_{2 \mathcal{R}} \\ \sqrt{c_{-}\left(1+N r_{ \pm}\right)} & \text {for } K=K_{1}, K_{2} \text { and } K_{3}\end{cases}
$$

acquires mass, at the SUSY vacuum in Eq. (19), which is given by

$$
\frac{\widehat{m}_{\delta \phi}}{\lambda m_{\mathrm{P}}}= \begin{cases}\sqrt{c_{\mathcal{R}}\left(N c_{\mathcal{R}}-1\right)} & \text { for } K=K_{1 \mathcal{R}} \text { and } K_{2 \mathcal{R}} \\ c_{-} \sqrt{2\left(1+N r_{ \pm}\right)} & \text {for } K=K_{1}, K_{2} \text { and } K_{3}\end{cases}
$$

From the last expression we can infer that $\widehat{m}_{\delta \phi}$ remains constant for fixed $n$ since $\lambda / c_{\mathcal{R}}\left[\lambda / c_{-}\right]$is fixed too-see Eqs. (48) and (58). More specifically, for the allowed range of $n$ in Eqs. (61a) and (61b) we obtain

$$
\begin{aligned}
& \text { 2. } \lesssim \widehat{m}_{\delta \phi} / 10^{13} \mathrm{GeV} \lesssim 3.9 \text { for } K=K_{1 \mathcal{R}} \text {, } \\
& 2.1 \lesssim \widehat{m}_{\delta \phi} / 10^{13} \mathrm{GeV} \lesssim 4.5 \quad \text { for } K=K_{2 \mathcal{R}} \text {, }
\end{aligned}
$$

with the value $\widehat{m}_{\delta \phi}=2.8 \cdot 10^{13} \mathrm{GeV}$ corresponding to $n=0$. Furthermore, for $K=K_{1}$ and the allowed range of $n$ in Eq. (64) we obtain

$2.9 \lesssim \widehat{m}_{\delta \phi} / 10^{13} \mathrm{GeV} \lesssim 5$

For $K=K_{2}$ and $K_{3}$, in the allowed ranges of Eqs. (65) and (66), we obtain

$3.1 \lesssim \widehat{m}_{\delta \phi} / 10^{13} \mathrm{GeV} \lesssim 6.9 ;$

$4.1 \lesssim \widehat{m}_{\delta \phi} / 10^{13} \mathrm{GeV} \lesssim 7.2$.

We remark that $\widehat{m}_{\delta \phi}$ is somewhat affected by the choice of $K$ 's in Eqs. (6a)-(6c). For $n=0, \widehat{m}_{\delta \phi}=4.7 \cdot 10^{13} \mathrm{GeV}$ for $K=K_{1}$, and $\widehat{m}_{\delta \phi}=5.2 \cdot 10^{13} \mathrm{GeV}$ for $K=K_{2}$ and $K_{3}$, which are both somewhat larger than the value obtained within Starobinsky inflation [4,5,10-12]. On the other hand, these values are close to the maximal ones found in Ref. [35], since here $r_{ \pm}$approaches its maximal value.

The inflaton can decay [106] perturbatively into:

(a) A pair of $N^{c_{i}}$ with Majorana masses $M_{i N^{c}}=\lambda_{i N^{c}} M$ with the decay width

$$
\widehat{\Gamma}_{\delta \phi \rightarrow N_{i}^{c} N_{i}^{c}}=\frac{g_{i N^{c}}^{2}}{16 \pi} \widehat{m}_{\delta \phi}\left(1-\frac{4 M_{i N^{c}}^{2}}{\widehat{m}_{\delta \phi}^{2}}\right)^{3 / 2},
$$

where the relevant coupling constant

$$
g_{i N^{c}}=(N-1) \frac{\lambda_{i N^{c}}}{\langle J\rangle}
$$

arises from the lagrangian term

$$
\begin{aligned}
\mathcal{L}_{\widehat{\delta \phi} \rightarrow N_{i}^{c} N_{i}^{c}} & =-\frac{1}{2} e^{K / 2 m_{\mathrm{P}}^{2}} W_{\mathrm{HI}, N_{i}^{c}} N_{i}^{c} N_{i}^{c} N_{i}^{c}+\text { h.c. } \\
& =g_{i N^{c}} \widehat{\delta \phi} N_{i}^{c} N_{i}^{c}+\text { h.c. }
\end{aligned}
$$


This decay channel activates the mechanism of nTL, as sketched in Sect. 3.3.2.

(b) Higgses $H_{u}$ and $H_{d}$ with the decay width

$\widehat{\Gamma}_{\delta \phi \rightarrow H_{u} H_{d}}=\frac{2}{8 \pi} g_{H}^{2} \widehat{m}_{\delta \phi}$ where $g_{H}=\frac{\lambda_{\mu}}{\sqrt{2}}$

arises from the lagrangian term

$$
\begin{aligned}
\mathcal{L}_{\widehat{\delta \phi} \rightarrow H_{u} H_{d}} & =-e^{K / m_{\mathrm{P}}^{2}} K^{S S^{*}}\left|W_{\mathrm{HI}, S}\right|^{2} \\
& =-g_{H} \widehat{m}_{\delta \phi} \widehat{\delta \phi}\left(H_{u}^{*} H_{d}^{*}+\text { h.c. }\right)+\cdots
\end{aligned}
$$

Thanks to the upper bounds on $\lambda_{\mu}$ from Eqs. (70c) and (70d), $g_{H}$ turns out to be comparable with $g_{i N^{c}}$.

(c) MSSM (s)-particles $X Y Z$ with the following $c_{+}$ -dependent 3-body decay width

$\widehat{\Gamma}_{\delta \phi \rightarrow X Y Z}=g_{y}^{2} \frac{n_{\mathrm{f}}}{512 \pi^{3}} \frac{\widehat{m}_{\delta \phi}^{3}}{m_{\mathrm{P}}^{2}}$,

where for the third generation we take $y \simeq(0.4-0.6)$, computed at the $\widehat{m}_{\delta \phi}$ scale, and $n_{\mathrm{f}}=14$ for $\widehat{m}_{\delta \phi}<$ $M_{3 N^{c}}$. Also,

$g_{y}=y_{3} \cdot \begin{cases}\sqrt{\left(N c_{\mathcal{R}}-1\right) / 2 c_{\mathcal{R}}} & \text { for } K=K_{1 \mathcal{R}} \text { and } K_{2 \mathcal{R}} \\ N \sqrt{r_{ \pm} /\left(1+N r_{ \pm}\right)} & \text {for } K=K_{1}, K_{2} \text { and } K_{3}\end{cases}$

and $y_{3}=h_{t, b, \tau}\left(\widehat{m}_{\delta \phi}\right) \simeq 0.5$. Since $r_{ \pm} \simeq 1 / N$ we can easily infer that $g_{y}$ above is enhanced compared to the corresponding one in Ref. [35] where $r_{ \pm} \simeq 0.01$, and an additional suppression through a ratio $M / m_{\mathrm{P}}$ exists. We therefore expect that $\widehat{\Gamma}_{\delta \phi \rightarrow X Y Z}$ contributes sizably to the total decay width of $\widehat{\delta \phi}$. Each individual decay width arises from the langrangian terms

$$
\begin{aligned}
\mathcal{L}_{\widehat{\delta \phi} \rightarrow X \psi_{Y} \psi_{Z}} & =-\frac{1}{2} e^{K / 2 m_{\mathrm{P}}^{2}}\left(W_{y, Y Z} \psi_{Y} \psi_{Z}\right)+\text { h.c. } \\
& =-g_{y} \frac{\widehat{\delta \phi}}{m_{\mathrm{P}}}\left(X \psi_{Y} \psi_{Z}\right)+\text { h.c. }
\end{aligned}
$$

where $W_{y}=y X Y Z$ is a typical trilinear superpotential term of MSSM with $y$ a Yukawa coupling constant, and $\psi_{X}, \psi_{Y}$ and $\psi_{Z}$ are the chiral fermions associated with the superfields $X, Y$ and $Z$ whose scalar components are denoted with the superfield symbols.

The resulting reheat temperature is given by [107]

$$
T_{\mathrm{rh}}=\left(72 / 5 \pi^{2} g_{*}\right)^{1 / 4} \widehat{\Gamma}^{1 / 2} m_{\mathrm{P}}^{1 / 2}
$$

with the total decay width of $\widehat{\delta \phi}$ being

$\widehat{\Gamma}=\widehat{\Gamma}_{\delta \phi \rightarrow N_{i}^{c} N_{i}^{c}}+\widehat{\Gamma}_{\delta \phi \rightarrow H_{u} H_{d}}+\widehat{\Gamma}_{\delta \phi \rightarrow X Y Z}$.

Here, $g_{*}=228.75$ counts the MSSM effective number of relativistic degrees of freedom at temperature $T_{\mathrm{rh}}$. Let us clarify here that in our models there is no decay of a scalaron as in the original (non-SUSY) [3] Starobinsky inflation and some [108-111] of its SUGRA realizations; thus, $T_{\text {rh }}$ in our case is slightly lower than that obtained there.

\subsubsection{Lepton-number and gravitino abundances}

For $T_{\mathrm{rh}}<M_{i N^{c}}$, the out-of-equilibrium decay of $v_{i}^{c}$ generates a lepton-number asymmetry (per $v_{i}^{c}$ decay), $\varepsilon_{i}$ - see, e.g., Refs. [64-69]. The resulting $\varepsilon_{i}$ is partially converted through sphaleron effects into a yield of the observed BAU [35, 64-69],

$Y_{B}=-0.35 \cdot 2 \cdot \frac{5}{4} \frac{T_{\mathrm{rh}}}{\widehat{m}_{\delta \phi}} \sum_{i} \frac{\widehat{\Gamma}_{\delta \phi \rightarrow N_{i}^{c} N_{i}^{c}}}{\widehat{\Gamma}} \varepsilon_{i}$

which has to reproduce the observational result [97]

$Y_{B}=\left(8.64_{-0.16}^{+0.15}\right) \cdot 10^{-11}$.

The validity of Eq. (87) requires that the $\widehat{\delta \phi}$ decay into a pair of $N_{i}^{c}$ 's is kinematically allowed for at least one species of the $N_{i}^{c}$ 's and also that there is no erasure of the produced $Y_{L}$ due to $N_{1}^{c}$ mediated inverse decays and $\Delta L=1$ scatterings [113]. These prerequisites are ensured if we impose

(a) $\widehat{m}_{\delta \phi} \geq 2 M_{1 N^{c}}$ and (b) $M_{1 N^{c}} \gtrsim 10 T_{\mathrm{rh}}$.

The quantity $\varepsilon_{i}$ can be expressed in terms of the Dirac masses of $v_{i}, m_{i \mathrm{D}}$, arising from the second term of Eq. (67a) see Ref. [35]. Moreover, employing the seesaw formula we can then obtain the light-neutrino mass matrix $m_{v}$ in terms of $m_{i \mathrm{D}}$ and $M_{i N^{c}}$. As a consequence, nTL can be nicely linked to low energy neutrino data. We take as inputs the best-fit values [81] - see also Refs. [82,83] - of the neutrino mass-squared differences, $\Delta m_{21}^{2}=7.6 \cdot 10^{-5} \mathrm{eV}^{2}$ and $\Delta m_{31}^{2}=(2.48[-2.38]) \cdot 10^{-3} \mathrm{eV}^{2}$, of the mixing angles, $\sin ^{2} \theta_{12}=0.323, \sin ^{2} \theta_{13}=0.0226\left[\sin ^{2} \theta_{13}=0.029\right]$ and $\sin ^{2} \theta_{23}=0.567\left[\sin ^{2} \theta_{23}=0.573\right]$, and of the CP-violating Dirac phase $\delta=1.41 \pi[\delta=1.48 \pi]$ for normal [inverted] ordered (NO [IO]) neutrino masses, $m_{i v}$ 's. The sum of $m_{i v}$ 's is bounded from above by the data [97],

$\sum_{i} m_{i v} \leq 0.23 \mathrm{eV}$ 
at $95 \%$ c.l. This is more restrictive than the $90 \%$ c.l. upper bound arising from the effective electron neutrino mass in $\beta$-decay [114]:

$m_{\beta} \leq(0.061-0.165) \mathrm{eV}$,

where the range accounts for nuclear matrix element uncertainties.

The required $T_{\text {rh }}$ in Eq. (87) must be compatible with constraints on the $\widetilde{G}$ abundance, $Y_{3 / 2}$, at the onset of nucleosynthesis (BBN), which are [75-78] given approximately by

$Y_{3 / 2} \lesssim\left\{\begin{array}{l}10^{-14} \\ 10^{-13} \\ 10^{-12}\end{array}\right.$ for $m_{3 / 2} \simeq\left\{\begin{array}{l}0.69 \mathrm{TeV}, \\ 10.6 \mathrm{TeV}, \\ 13.5 \mathrm{TeV} .\end{array}\right.$

Here we consider the conservative case where $\widetilde{G}$ decays with a tiny hadronic branching ratio. The bounds above can be somehow relaxed in the case of a stable $\widetilde{G}-$ see e.g. Ref. [46].

In our models $Y_{3 / 2}$ is estimated to be [72-78]:

$Y_{3 / 2} \simeq 1.9 \cdot 10^{-22} T_{\mathrm{rh}} / \mathrm{GeV}$,

where we take into account only thermal production of $\widetilde{G}$, and assume that $\widetilde{G}$ is much heavier than the MSSM gauginos. Non-thermal contributions to $Y_{3 / 2}$ [106] are also possible but strongly dependent on the mechanism of soft SUSY breaking. Moreover, no precise computation of this contribution exists within IGHI adopting the simplest Polonyi model of SUSY breaking $[79,80]$. It is notable, though, that the nonthermal contribution to $Y_{3 / 2}$ in models with stabilizer field, as in our case, is significantly suppressed compared to the thermal one.

\subsubsection{Results}

It is worthwhile to test the applicability of the framework above in the case of IGHI. Namely, following a bottom-up approach detailed in Ref. [35], we find the $M_{i N^{c}}$ 's by using as inputs the $m_{i \mathrm{D}}$ 's, a reference mass of the $v_{i}$ 's $-m_{1 v}$ for NO $m_{i v}$ 's, or $m_{3 v}$ for IO $m_{i v}$ 's -, the two Majorana phases $\varphi_{1}$ and $\varphi_{2}$ of the PMNS matrix, and the best-fit values, mentioned in Sect. 3.3.2, for the low energy parameters of neutrino physics - note that there are no experimental constraints on $\varphi_{1}$ and $\varphi_{2}$ up to now. In our numerical code we also estimate, following Ref. [112], the renormalization group evolved values of the latter parameters at the scale of nTL, $\Lambda_{L}=\widehat{m}_{\delta \phi}$, by considering the MSSM with $\tan \beta \simeq 50$ as an effective theory between $\Lambda_{L}$ and the soft SUSY breaking scale, $M_{\text {SUSY }}=1.5 \mathrm{TeV}$. We evaluate the $M_{i N^{c}}$ 's at $\Lambda_{L}$, and we neglect any possible running of the $m_{i \mathrm{D}}$ 's and $M_{i N^{c}}$ 's. Therefore, we present their values at $\Lambda_{L}$.
We start the exposition of our results arranging in Table 6 for $K=K_{2}$ or $K_{3}$ and 7 for $K=K_{2 \mathcal{R}}$ some representative values of the parameters which yield $Y_{B}$ and $Y_{3 / 2}$ compatible with Eqs. (88) and (92), respectively. Throughout our computation we take $\lambda_{\mu}=10^{-6}$, in accordance with Eqs. (70c) and (70d), and $y=0.5$, which is a typical value encountered [100] in various MSSM settings with large $\tan \beta$. Also, we select $n=0.02$ in Table 6 and $n=0$ in Table 7 . These values yield $n_{\mathrm{s}}$ and $r$ in the "sweet" spot of the present data - see Figs. 1 and 2. We obtain $M=2.85 \cdot 10^{16} \mathrm{GeV}$ and $\widehat{m}_{\delta \phi}=$ $2.8 \cdot 10^{13} \mathrm{GeV}$ for $K=K_{1 \mathcal{R}}$ or $K_{2 \mathcal{R}}, M=6.1 \cdot 10^{17} \mathrm{GeV}$ and $\widehat{m}_{\delta \phi}=4.2 \cdot 10^{13} \mathrm{GeV}$ for $K=K_{1}$, or $M=5.6 \cdot 10^{15} \mathrm{GeV}$ and $\widehat{m}_{\delta \phi}=4.4 \cdot 10^{13} \mathrm{GeV}$ for $K=K_{2}$ or $K_{3}$. Although the uncertainties from the choice of $K$ 's are negligible as regards the quantities above, the decay widths in Sect. 3.3.1 depend on $N$ (and $r_{ \pm}$) which take slightly different values for $K=K_{1 \mathcal{R}}$ or $K_{1}$ and $K=K_{2 \mathcal{R}}, K_{2}$ or $K_{3}$ - see e.g. Fig. 3 - discriminating somehow the various choices. For this reason, we clarify that we adopt $K=K_{2 \mathcal{R}}$ in Table 7 and $K=K_{2}$ or $K_{3}$ in Table 6. Had we employed $K=K_{1 \mathcal{R}}$ or $K_{1}$, we would have obtained almost two times larger $Y_{B}$ 's with the same values of the free parameters. Therefore a mild readjustment is needed.

In both Tables we consider NO (cases A and B), almost degenerate (cases $\mathrm{C}, \mathrm{D}$ and $\mathrm{E}$ ) and IO (cases F and G) $m_{i v}$ 's. In all cases Eq. (90) is safely met - the case D saturates it - whereas Eq. (91) is comfortably satisfied. The gauge group adopted here, $G_{B-L}$, does not predict any relation between the Yukawa couplings constants $h_{i N}$ entering the second term of Eq. (67a) and the other Yukawa couplings in the MSSM. As a consequence, the $m_{i \mathrm{D}}$ 's are free parameters. However, for the sake of comparison, for cases $\mathrm{A}-\mathrm{F}$, we take $m_{3 \mathrm{D}}=m_{t}\left(\Lambda_{L}\right) \simeq 100 \mathrm{GeV}$, where $m_{t}$ denotes the mass of the top quark. Similar conditions for the lighter generations do not hold, though, in our data sample.

Besides case A, where only the channel $\widehat{\delta \phi} \rightarrow N_{1}^{c} N_{1}^{c}$ is kinematically unblocked, $\widehat{\delta \phi}$ decays into $N_{1}^{c}$ 's and $N_{2}^{c}$ 's. In the latter cases $\varepsilon_{2}$ yields the dominant contribution to the calculation $Y_{B}$ from Eq. (87). From our computation, we also remark that $\widehat{\Gamma}_{\delta \phi \rightarrow N_{i}^{c} N_{i}^{c}}<\widehat{\Gamma}_{\delta \phi \rightarrow H_{u} H_{d}}<\widehat{\Gamma}_{\delta \phi \rightarrow X Y Z}$, and so the ratios $\widehat{\Gamma}_{\delta \phi \rightarrow N_{i}^{c}} N_{i}^{c} / \widehat{\Gamma}$ introduce a considerable reduction $(0.02-0.25)$ in the derivation of $Y_{B}$. As a consequence, the attainment of the correct $Y_{B}$ requires relatively large $m_{i \mathrm{D}}$ 's

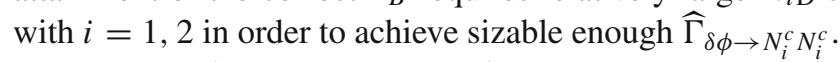
Namely, $m_{1 \mathrm{D}} \gtrsim 1 \mathrm{GeV}$ and $m_{2 \mathrm{D}} \gtrsim 6.6 \mathrm{GeV}$. Besides case $\mathrm{A}$, the first inequality is necessary, in order to fulfill the second inequality in Eq. (89), given that $m_{1 \mathrm{D}}$ heavily influences $M_{1 N^{c}}$. In Table 7 we list only $m_{1 \mathrm{D}}$ in case A or $m_{2 \mathrm{D}}$ in the other cases which are adjusted so as to accommodate $Y_{B}$ within the range of Eq. (88) with the others $m_{i \mathrm{D}}$ remaining as shown in Table 6 . As a consequence, $M_{i N^{c}}$ deviate very little from the values shown in Table 6. 
Table 6 Parameters yielding the correct BAU for $K=K_{2}$ or $K_{3}, n=0.02, \lambda_{\mu}=10^{-6}$, $y_{3}=0.5$ and various neutrino mass schemes

\begin{tabular}{|c|c|c|c|c|c|c|c|}
\hline \multirow[t]{3}{*}{ Parameters } & \multicolumn{7}{|l|}{ Cases } \\
\hline & A & $\mathrm{B}$ & \multirow[t]{2}{*}{$\mathrm{C}$} & $\mathrm{D}$ & $\mathrm{E}$ & $\mathrm{F}$ & G \\
\hline & \multicolumn{2}{|c|}{$\begin{array}{c}\text { Normal } \\
\text { hierarchy }\end{array}$} & & \multicolumn{2}{|c|}{$\begin{array}{c}\text { Almost } \\
\text { degeneracy }\end{array}$} & \multicolumn{2}{|c|}{$\begin{array}{l}\text { Inverted } \\
\text { hierarchy }\end{array}$} \\
\hline \multicolumn{8}{|c|}{ Low scale parameters (masses in $\mathrm{eV}$ ) } \\
\hline$m_{1 v} / 0.1$ & 0.01 & 0.1 & 0.5 & 0.7 & 0.7 & 0.5 & 0.49 \\
\hline$m_{2 v} / 0.1$ & 0.09 & 0.13 & 0.51 & 1.0 & 0.705 & 0.51 & 0.5 \\
\hline$m_{3 v} / 0.1$ & 0.5 & 0.51 & 0.71 & 1.12 & 0.5 & 0.1 & 0.05 \\
\hline$\sum_{i} m_{i v} / 0.1$ & 0.6 & 0.74 & 1.7 & 2.3 & 1.9 & 1.1 & 1 \\
\hline$m_{\beta} / 0.01$ & 0.22 & 0.98 & 3.5 & 5.3 & 2.9 & 4.9 & 3.6 \\
\hline$\varphi_{1}$ & 0 & 0 & 0 & $\pi / 2$ & $\pi / 2$ & $-3 \pi / 4$ & 0 \\
\hline$\varphi_{2}$ & $-\pi / 2$ & 0 & $\pi / 2$ & $-\pi$ & $-2 \pi / 3$ & $5 \pi / 4$ & $-\pi / 2$ \\
\hline \multicolumn{8}{|c|}{ Leptogenesis-scale mass parameters in $\mathrm{GeV}$} \\
\hline$m_{1 \mathrm{D}}$ & 1.98 & 1.5 & 2.3 & 4.16 & 5.2 & 1 & 6.3 \\
\hline$m_{2 \mathrm{D}}$ & 38 & 16.6 & 12 & 10 & 9.6 & 6.6 & 10 \\
\hline$m_{3 \mathrm{D}} / 100$ & 1 & 1 & 1 & 1 & 1 & 1 & 0.33 \\
\hline$M_{1 N^{c}} / 10^{11}$ & 1.6 & 2.1 & 1.4 & 2.8 & 5.2 & 0.2 & 8.9 \\
\hline$M_{2 N^{c}} / 10^{12}$ & 27 & 6.8 & 2.6 & 4.8 & 1.9 & 2.2 & 3.2 \\
\hline$M_{3 N^{c}} / 10^{14}$ & 22 & 4.7 & 0.89 & 0.22 & 0.69 & 2.9 & 0.9 \\
\hline \multicolumn{8}{|c|}{ Decay channels of the inflaton $\widehat{\delta \phi}$} \\
\hline$\widehat{\delta \phi} \rightarrow$ & $N_{1}^{c}$ & $N_{1,2}^{c}$ & $N_{1,2}^{c}$ & $N_{1,2}^{c}$ & $N_{1,2}^{c}$ & $N_{1,2}^{c}$ & $N_{1,2}^{c}$ \\
\hline \multicolumn{8}{|c|}{ Resulting $B$-Yield } \\
\hline$Y_{B}^{0} / 10^{-11}$ & 9.63 & 8 & 8.4 & 9.1 & 8.9 & 8.7 & 8.9 \\
\hline$Y_{B} / 10^{-11}$ & 8.67 & 8.59 & 8.69 & 8.56 & 8.65 & 8.67 & 8.65 \\
\hline \multicolumn{8}{|c|}{ Resulting $T_{\text {rh }}$ (in $\mathrm{GeV}$ ) and $\widetilde{G}$-Yield } \\
\hline$T_{\mathrm{rh}} / 10^{9}$ & 1 & 1.1 & 1 & 1.1 & 1 & 1 & 1 \\
\hline $10^{13} Y_{3 / 2}$ & 1.91 & 2.2 & 1.9 & 2 & 1.9 & 1.9 & 1.97 \\
\hline
\end{tabular}

Table 7 Same as in Table 6 but for $K=K_{2 \mathcal{R}}$ and $n=0$

\begin{tabular}{lllllllll}
\hline Parameters & \multicolumn{2}{l}{ Cases } & & & & & & \\
\cline { 2 - 7 } & A & B & C & D & E & F & G \\
\hline
\end{tabular}

Low scale parameters as in Table 6

Leptogenesis-scale mass parameters in $\mathrm{GeV}$

\begin{tabular}{|c|c|c|c|c|c|c|}
\hline$m_{i \mathrm{D}}^{(*)}$ & 1.91 & 16.6 & 11.6 & 10.15 & 9.25 & 6.37 \\
\hline \multicolumn{7}{|c|}{ Resulting $B$-Yield } \\
\hline$Y_{B}^{0} / 10^{-11}$ & 9.6 & 7.8 & 8.5 & 8.9 & 8.9 & 8.9 \\
\hline$Y_{B} / 10^{-11}$ & 8.64 & 8.61 & 8.72 & 8.6 & 8.73 & 8.8 \\
\hline
\end{tabular}

Resulting $T_{\text {rh }}$ (in $\mathrm{GeV}$ ) and $\widetilde{G}$-Yield

\begin{tabular}{llllllll}
$T_{\mathrm{rh}} / 10^{8}$ & 7.6 & 8.4 & 7.7 & 8.4 & 7.6 & 7.6 & 7.8 \\
$Y_{3 / 2} / 10^{-13}$ & 1.44 & 2.9 & 1.5 & 1.6 & 1.45 & 1.45 & 1.5 \\
\hline
\end{tabular}

(*) Where $i=1$ for case A and $i=2$ for the others; the remaining $m_{i \mathrm{D}}$ and $m_{i} N_{c}$ are as in Table 6

In both Tables we also display, for comparison, the $B$-yield with $\left(Y_{B}\right)$ or without $\left(Y_{B}^{0}\right)$ taking into account the renormalization group effects. We observe that the two results are mostly close to each other. Shown also are values for $T_{\mathrm{rh}}$, the majority of which are close to $10^{9} \mathrm{GeV}$, and the corresponding $Y_{3 / 2}$ 's, with the results for $K=K_{2 \mathcal{R}}$ being a little lower. Thanks to our non-thermal set-up, successful leptogenesis can be accommodated with $T_{\text {rh }}$ 's lower than those necessitated in the thermal regime - cf. Ref. [115]. The resulting large $Y_{3 / 2}$ 's may be consistent with Eq. (92) mostly for $m_{3 / 2} \gtrsim 10 \mathrm{TeV}$. These are marginally tolerated with the $m_{3 / 2}$ 's appearing in Table 5 and Figs. 2 and 3 of Ref. [104] in the $A / H$ funnel and $\chi_{1}^{ \pm}-\chi$ coannihilation regions - see also Ref. [116]. These $m_{3 / 2}$ 's though are more easily reconciled with low energy data in less restrictive versions of MSSM - see e.g. Ref. [117].

In order to extend the conclusions inferred from Table 6 to the case of variable $n$, we can examine how the central value of $Y_{B}$ in Eq. (88) can be achieved by varying $m_{2 \mathrm{D}}$ as a function of $n$. The resulting contours in the $n-m_{2 \mathrm{D}}$ plane are presented in Fig. 6 - since the range of $Y_{B}$ in Eq. (88) is very narrow, the $95 \%$ c.l. width of these contours is negligible. The convention adopted for these lines is also described in the figure. In particular, we use solid, dashed, or 


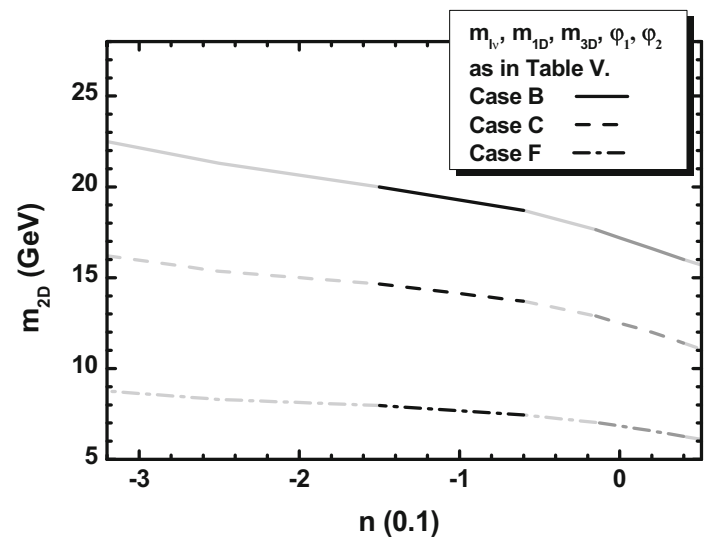

Fig. 6 Contours in the $n-m_{2}$ D plane yielding the central $Y_{B}$ in Eq. (88) consistently with the inflationary requirements for $K=K_{2}$ or $K_{3}$, $\lambda_{\mu}=10^{-6}, y_{3}=0.5$ and the values of $m_{i v}, m_{1 \mathrm{D}}, m_{3 \mathrm{D}}, \varphi_{1}$, and $\varphi_{2}$ which correspond to the cases $\mathrm{B}$ (solid line), $\mathrm{C}$ (dashed line), and $\mathrm{F}$ (dot-dashed line) of Table 6. The color coding is as in Fig. 3.

dot-dashed line for $m_{i v}, m_{1 \mathrm{D}}, m_{3 \mathrm{D}}, \varphi_{1}$, and $\varphi_{2}$ corresponding to the cases B, D, or F of Table 6 respectively. For $n$ within its allowed margins in Eqs. (65) and (66) we obtain $0.4 \lesssim$ $T_{\mathrm{rh}} / 10^{9} \mathrm{GeV} \lesssim 1.8$, which is perfectly acceptable from Eq. (92) for $m_{3 / 2} \gtrsim 10 \mathrm{TeV}$. Along the depicted contours, the resulting $M_{2 N^{c}}$ 's vary in the ranges $(5.7-14.5) \cdot 10^{12} \mathrm{GeV}$, $(1.8-4.6) \cdot 10^{12} \mathrm{GeV},(1.5-3.9) \cdot 10^{12} \mathrm{GeV}$ for cases B, C and $\mathrm{F}$ respectively, whereas $M_{1 N^{c}}$ and $M_{3 N^{c}}$ remain close to their values presented in the corresponding cases of Table 6 .

Comparing, finally, our results above with those presented in Ref. [35], we can deduce that here $\widehat{m}_{\delta \phi}$ and $T_{\text {rh }}$ gain almost their maximal allowed values since $r_{ \pm}$is also maximized due to the hypothesis of Eq. (38). As a consequence, $m_{3 / 2}$ also has to be enhanced to avoid problems with BBN, whereas $m_{1,2 \mathrm{D}}$ and $M_{1,2 N^{c}}$ are also constrained to larger values. On the other hand, our results are closer to those obtained employing the model of IG in Refs. [4,5] with gauge singlet inflaton and without unification constraint.

\section{Conclusions}

We have proposed a class of novel inflationary models, referred to as IGHI, in which a Higgs field plays the role of the inflaton, before settling in its final vacuum state where it generates the Planck scale and gives rise to a mass for the gauge boson consistent with gauge coupling unification within MSSM. These two hypotheses allow us to determine the mass scale $M$ entering $W_{\mathrm{HI}}$ in Eq. (1), and the quantity $c_{\mathcal{R}}$ for the $K$ 's in Eqs. (4a) and (4b) or $r_{ \pm}=c_{+} / c_{-}$for the $K$ 's in Eqs. (6a)-(6c). In the latter cases, $r_{ \pm}$expresses the amount of violation of a shift symmetry otherwise respected by these $K$ 's. As a consequence, the inflationary scenario depends essentially on two free parameters $-n$ and $\lambda$, for the first group of $K$ 's, or $\lambda / c_{-}$, in the second group of $K$ 's, - leading naturally to observationally acceptable results. Namely, for the $K$ 's in Eqs. (6a)-(6c) we obtained slightly larger $r$ 's and two distinct allowed regions of parameters with $n$ values one order of magnitude larger than those needed for the $K$ 's in Eqs. (4a) and (4b). As an example, the model for $K=K_{2}$ or $K_{3}, n=0$ and $\lambda / c_{-}=3 \cdot 10^{-5}$ yields $n_{\mathrm{s}} \simeq 0.973$ and $r \simeq 0.0066$ with negligibly small $a_{\mathrm{s}}$. In both groups of $K$ 's, IGHI is attained for subplanckian values of the inflaton, thereby stabilizing our predictions from possible higher order corrections in $W_{\mathrm{HI}}$ and/or $K$ 's. Moreover, the corresponding effective theories remain trustable up to $m_{\mathrm{P}}$.

The models were further extended to generate the MSSM $\mu$ parameter, consistently with the low energy phenomenology. Successful baryogenesis is achieved via primordial leptogenesis, in agreement with the data on neutrino masses and mixing. More specifically, our post-inflationary setting favors the $A$ / $H$ funnel and the $\tilde{\chi}_{1}^{ \pm}-\chi$ coannihilation regions of CMSSM with gravitino heavier than about $10 \mathrm{TeV}$. Moreover, leptogenesis is realized through the out-of equilibrium decay of the inflaton to the right-handed neutrinos $N_{1}^{c}$ and/or $N_{2}^{c}$, with masses lower than $3.5 \cdot 10^{13} \mathrm{GeV}$, and a reheat temperature $T_{\text {rh }}$ close to $10^{9} \mathrm{GeV}$.

Acknowledgements C.P. acknowledges the Bartol Research Institute and the Department of Physics and Astronomy of the University of Delaware for its warm hospitality, during which this work has been initiated. He also acknowledges useful discussions with G. Lazarides and S. Martin. Q.S. acknowledges support by the DOE Grant no. DESC0013880.

Open Access This article is distributed under the terms of the Creative Commons Attribution 4.0 International License (http://creativecomm ons.org/licenses/by/4.0/), which permits unrestricted use, distribution, and reproduction in any medium, provided you give appropriate credit to the original author(s) and the source, provide a link to the Creative Commons license, and indicate if changes were made. Funded by SCOAP ${ }^{3}$.

\section{References}

1. A. Zee, Phys. Rev. Lett 42, 417 (1979)

2. H. Terazawa, Phys. Lett. B 101, 43 (1981)

3. A.A. Starobinsky, Phys. Lett. B 91, 99 (1980)

4. C. Pallis, J. Cosmol. Astropart. Phys 04, 024 (2014) [Erratum: C. Pallis, J. Cosmol. Astropart. Phys 07, 01(E) (2017)]. arXiv: 1312.3623

5. C. Pallis, J. Cosmol. Astropart. Phys 07, 01 (2017). arXiv: 1312.3623

6. R. Kallosh, Phys. Rev. D 89, 087703 (2014). arXiv:1402.3286

7. C. Pallis, J. Cosmol. Astropart. Phys 08, 057 (2014). arXiv:1403.5486D

8. C. Pallis, J. Cosmol. Astropart. Phys 10, 058 (2014). arXiv: 1407.8522

9. C. Pallis, PoS CORFU 2014, 156 (2015). arXiv:1506.03731

10. C. Pallis, N. Toumbas, J. Cosmol, Astropart. Phys 05(05), 015 (2016). arXiv:1512.05657 
11. C. Pallis, N. Toumbas, Adv. High Energy Phys 2017, 6759267 (2017). arXiv: 1612.09202

12. C. Pallis, PoS EPS-HEP 2017, 047 (2017). arXiv:1710.04641

13. M.B. Einhorn, D.R.T. Jones, J. Cosmol. Astropart. Phys 11, 049 (2012). arXiv:1207.1710

14. F.S. Accetta, D.J. Zoller, M.S. Turner, Phys. Rev. D 31, 3046 (1985)

15. R. Fakir, W.G. Unruh, Phys. Rev. D 41, 1792 (1990)

16. D.I. Kaiser, Phys. Rev. D 52, 4295 (1995). arXiv:astro-ph/9408044

17. D.S. Salopek, J.R. Bond, J.M. Bardeen, Phys. Rev. D 40, 1753 (1989)

18. J.L. Cervantes-Cota, H. Dehnen, Phys. Rev. D 51, 395 (1995). arXiv:astro-ph/9412032

19. N. Kaloper, L. Sorbo, J. Yokoyama, Phys. Rev. D 78, 043527 (2008). arXiv:0803.3809

20. G.F. Giudice, H.M. Lee, Phys. Lett. B 733, 58 (2014). arXiv: 1402.2129

21. K. Kannike et al., J. High Energy Phys 05, 065 (2015). arXiv: 1502.01334

22. M.B. Einhorn, D.R.T. Jones, J. High Energy Phys 01, 019 (2016). arXiv: 1511.01481

23. P.A.R. Ade et al. [Planck Collaboration], Astron. Astrophys 594, A20 (2016). arXiv: 1502.02114

24. J.L.F. Barbon, J.R. Espinosa, Phys. Rev. D 79, 081302 (2009). arXiv:0903.0355

25. C.P. Burgess, H.M. Lee, M. Trott, J. High Energy Phys 07, 007 (2010). arXiv:1002.2730

26. A. Kehagias, A.M. Dizgah, A. Riotto, Phys. Rev. D 89, 043527 (2014). arXiv:1312.1155

27. C. Pallis, Phys. Lett. B 692, 287 (2010). arXiv: 1002.4765

28. R. Kallosh, A. Linde, D. Roest, Phys. Rev. Lett 112, 011303 (2014). arXiv: 1310.3950

29. N. Okada, M.U. Rehman, Q. Shafi, Phys. Rev. D 82, 043502 (2010). arXiv: 1005.5161

30. C. Pallis, N. Toumbas, J. Cosmol. Astropart. Phys 12, 002 (2011). arXiv: 1108.1771

31. C. Pallis, N. Toumbas, Open questions in cosmology (InTech, 2012). arXiv: 1207.3730

32. G. Lazarides, C. Pallis, J. High Energy Phys 11, 114 (2015). arXiv: 1508.06682

33. C. Pallis, Phys. Rev. D 92(12), 121305(R) (2015). arXiv: 1511.01456

34. C. Pallis, J. Cosmol. Astropart. Phys 10, 03710 (2016). arXiv: 1606.09607

35. C. Pallis, Universe 4(1), 13 (2018). arXiv: 1510.05759

36. G.R. Dvali, Q. Shafi, R.K. Schaefer, Phys. Rev. Lett 73, 1886 (1994). arXiv:hep-ph/9406319

37. G.R. Dvali, G. Lazarides, Q. Shafi, Phys. Lett. B 424, 259 (1998). arXiv:hep-ph/9710314

38. M. Bastero-Gil, S.F. King, Q. Shafi, Phys. Lett. B 651, 345 (2007). arXiv:hep-ph/0604198

39. B. Garbrecht, C. Pallis, A. Pilaftsis, J. High Energy Phys 12, 038 (2006). arXiv:hep-ph/0605264

40. M.U. Rehman, V.N. Şenoğuz, Q. Shafi, Phys. Rev. D 75, 043522 (2007). arXiv:hep-ph/0612023

41. C. Pallis, J. Cosmol. Astropart. Phys 04, 024 (2009). arXiv:0902.0334

42. M. Civiletti, C. Pallis, Q. Shafi, Phys. Lett. B 733, 276 (2014). arXiv: 1402.6254

43. V.N. Şenoğuz, Q. Shafi. arXiv:hep-ph/0512170

44. W. Buchmüller, V. Domcke, K. Schmitz, Nucl. Phys B862, 587 (2012). arXiv: 1202.6679

45. C. Pallis, Q. Shafi, Phys. Lett. B 725, 327 (2013). arXiv:1304.5202

46. N. Okada, Q. Shafi, Phys. Lett. B 775, 348 (2017). arXiv: 1506.01410
47. N. Okada, Q. Shafi. arXiv:1709.04610

48. M. Kawasaki, M. Yamaguchi, T. Yanagida, Phys. Rev. Lett 85, 3572 (2000). arXiv:hep-ph/0004243

49. P. Brax, J. Martin, Phys. Rev. D 72, 023518 (2005). arXiv:hep-th/0504168

50. S. Antusch, K. Dutta, P.M. Kostka, Phys. Lett. B 677, 221 (2009). arXiv:0902.2934

51. R. Kallosh, A. Linde, T. Rube, Phys. Rev. D 83, 043507 (2011). arXiv: 1011.5945

52. T. Li, Z. Li, D.V. Nanopoulos, J. Cosmol. Astropart. Phys 02, 028 (2014). arXiv:1311.6770

53. K. Harigaya, T.T. Yanagida, Phys. Lett. B 734, 13 (2014) arXiv: 1403.4729

54. A. Mazumdar, T. Noumi, M. Yamaguchi, Phys. Rev. D 90, 043519 (2014). arXiv:1405.3959

55. C. Pallis, Q. Shafi, Phys. Lett. B 736, 261 (2014). arXiv:1405.7645

56. I. Ben-Dayan, M.B. Einhorn, J. Cosmol. Astropart. Phys 12, 002 (2010). arXiv:1009.2276

57. C. Pallis, Phys. Rev. D 91, 12350812 (2015). arXiv: 1503.05887

58. C. Pallis, PoS PLANCK 2015, 095 (2015). arXiv:1510.02306

59. P.A.R. Ade et al. [BICEP $2 /$ Keck Array Collaborations], Phys. Rev. Lett 116, 031302 (2016). arXiv: 1510.09217

60. W.L.K. Wu et al., J. Low. Temp. Phys 184(3-4), 765 (2016). arXiv: 1601.00125

61. P. Andre et al. [PRISM Collaboration]. arXiv:1306.2259

62. T. Matsumura et al., J. Low Temp. Phys. 176, 733 (2014). arXiv: 1311.2847

63. F. Finelli et al. [CORE Collaboration]. arXiv:1612.08270

64. K. Hamaguchi, Phd Thesis. arXiv:hep-ph/0212305

65. W. Buchmuller, R.D. Peccei, T. Yanagida, Ann. Rev. Nucl. Part. Sci 55, 311 (2005). arXiv:hep-ph/0502169

66. G. Lazarides, Q. Shafi, Phys. Lett. B 258, 305 (1991)

67. K. Kumekawa, T. Moroi, T. Yanagida, Prog. Theor. Phys 92, 437 (1994). arXiv:hep-ph/9405337

68. G. Lazarides, R.K. Schaefer, Q. Shafi, Phys. Rev. D 56, 1324 (1997). arXiv:hep-ph/9608256

69. V.N. Şenoğuz, Q. Shafi, Phys. Rev. D 71, 043514 (2005). arXiv:hep-ph/0412102

70. M. Yu, A.D.Linde Khlopov, Phys. Lett. B 265, 138 (1984)

71. J. Ellis, J.E. Kim, D.V. Nanopoulos, Phys. Lett. B 145, 181 (1984)

72. M. Bolz, A. Brandenburg, W. Buchmüller, Nucl. Phys. B B606, 518 (2001) [Erratum: M. Bolz, A. Brandenburg, W. Buchmuller, Nucl. Phys. 790, 336(E) (2008)]. arXiv:hep-ph/0012052

73. M. Bolz, A. Brandenburg, W. Buchmüller, Nucl. Phys 790, 336(E) (2008). arXiv:hep-ph/0012052

74. J. Pradler, F.D. Steffen, Phys. Rev. D 75, 023509 (2007). arXiv:hep-ph/0608344

75. M. Kawasaki, K. Kohri, T. Moroi, Phys. Lett. B 625, 7 (2005). arXiv:astro-ph/0402490

76. M. Kawasaki, K. Kohri, T. Moroi, Phys. Rev. D 71, 083502 (2005). arXiv:astro-ph/0408426

77. J.R. Ellis, K.A. Olive, E. Vangioni, Phys. Lett. B 619, 30 (2005). arXiv:astro-ph/0503023

78. M. Kawasaki, K. Kohri, T. Moroi, Y. Takaesu, Phys. Rev. D 97(2), 023502 (2018). arXiv:1709.01211

79. J. Ellis et al., J. Cosmol. Astropart. Phys 03, 00803 (2016). arXiv: 1512.05701

80. Y. Ema et al., J. High Energy Phys 11, 184 (2016). arXiv: 1609.04716

81. D.V. Forero, M. Tortola, J.W.F. Valle, Phys. Rev. D 90(9), 093006 (2014). arXiv: 1405.7540

82. M.C. Gonzalez-Garcia, M. Maltoni, T. Schwetz, J. High Energy Phys 11, 052 (2014). arXiv: 1409.5439

83. F. Capozzi et al., Nucl. Phys. B 908, 218 (2016). arXiv: 1601.07777 
84. M.B. Einhorn, D.R.T. Jones, J. High Energy Phys 03, 026 (2010). arXiv:0912.2718

85. H.M. Lee, J. Cosmol. Astropart. Phys 08, 003 (2010). arXiv: 1005.2735

86. S. Ferrara et al., Phys. Rev. D 83, 025008 (2011). arXiv: 1008.2942

87. C. Pallis, N. Toumbas, J. Cosmol. Astropart. Phys 02, 019 (2011). arXiv: 1101.0325

88. G. Lopes Cardoso, D. Lüst, T. Mohaupt, Nucl. Phys. B 432, 68 (1994). arXiv:hep-th/9405002

89. I. Antoniadis, E. Gava, K.S. Narain, T.R. Taylor, Nucl. Phys B432, 187 (1994). arXiv:hep-th/9405024

90. R. Kallosh, A. Linde, D. Roest, J. High Energy Phys 11, 198 (2013). arXiv:1311.0472

91. R. Kallosh, A. Linde, D. Roest, J. High Energy Phys. 08, 052 (2014). arXiv:1405.3646

92. L. Boubekeur, D. Lyth, J. Cosmol. Astropart. Phys. 07, 010 (2005). arXiv:hep-ph/0502047

93. S.R. Coleman, E.J. Weinberg, Phys. Rev. D 719731888

94. D.H. Lyth, A. Riotto, Phys. Rept 314, 1 (1999). arXiv:hep-ph/9807278

95. J. Martin, C. Ringeval, V. Vennin, Phys. Dark Univ 5-6, 75 (2014). arXiv: 1303.3787

96. http://functions.wolfram.com

97. P.A.R. Ade et al. [Planck Collaboration], Astron. Astrophys 594, A13 (2016). arXiv:1502.01589

98. A. Racioppi. arxiv: 1801.08810

99. G. Lazarides, Q. Shafi, Phys. Rev. D 58, 071702 (1998). arXiv:hep-ph/9803397

100. S. Antusch, M. Spinrath, Phys. Rev. D 78, 075020 (2008). arXiv:0804.0717

101. W. Buchmüller et al., J. High Energy Phys. 09, 053 (2014). arXiv: 1407.0253
102. J. Ellis, M. Garcia, D. Nanopoulos, K. Olive, J. Cosmol. Astropart. Phys. 10, 003 (2015). arXiv:1503.08867

103. E. Dudas, T. Gherghetta, Y. Mambrini, K.A. Olive, Phys. Rev. D 96(11), 115032 (2017). arXiv:1710.07341

104. P. Athron, et al. [GAMBIT Collaboration], Eur. Phys. J. C 7782412(2017). arXiv: 1705.07935

105. A. Addazi, S.V. Ketov, M.Y. Khlopov, arXiv:1708.05393

106. M. Endo, F. Takahashi, T.T. Yanagida, Phys. Rev. D 76, 083509 (2007). arXiv:0706.0986

107. C. Pallis, Nucl. Phys. B B751, 129 (2006). arXiv:hep-ph/0510234

108. S.V. Ketov, A.A. Starobinsky, Phys. Rev. D 83, 063512 (2011). arXiv: 1011.0240

109. S.V. Ketov, N. Watanabe, J. Cosmol. Astropart. Phys. 03, 011 (2011). arXiv:1101.0450

110. S.V. Ketov, A.A. Starobinsky, J. Cosmol. Astropart. Phys. 08, 022 (2012). arXiv:1203.0805

111. S.V. Ketov, S. Tsujikawa, Phys. Rev. D 86, 023529 (2012). arXiv: 1205.2918

112. S. Antusch, J. Kersten, M. Lindner, M. Ratz, Nucl. Phys B674, 401 (2003). arXiv:hep-ph/0305273

113. V.N. Șenoğuz, Phys. Rev. D 76, 013005 (2007). arXiv:0704.3048

114. A. Gando et al. [KamLAND-Zen Collaboration], Phys. Rev. Lett 117(8), 082503 (2016). ibid17(10), 109903 (2016). arXiv: 1605.02889

115. S. Antusch, A.M. Teixeira, J. Cosmol. Astropart. Phys. 02, 024 (2007). arXiv:hep-ph/0611232

116. N. Karagiannakis, G. Lazarides, C. Pallis, Phys. Rev. D 92(8), 085018 (2015). arXiv:1503.06186

117. H. Baer et al., Phys. Rev. Lett. 109, 161802 (2012). arXiv:1207.3343 